\title{
An Immunohistochemical Panel of Three SUMO Genes Predicts Outcomes of Patients With Triple-negative Breast Cancer
}

\section{Yuxiang Lin}

Fujian Medical University Union Hospital

\section{Qingshui Wang}

Fujian Normal University

\section{Han Xiao}

Fujian Medical University Union Hospital

\section{Zhiwei Chen}

Fujian Provincial Maternity and Children's Hospital: Fujian Provincial Maternity and Children's Hospital

\section{Meichen Jiang}

Fujian Medical University Union Hospital

Jie Zhang

Fujian Medical University Union Hospital

\section{Rongrong Guo}

Fujian Medical University Union Hospital

\section{Shaohong Kang}

Fujian Medical University Union Hospital

Yao Lin

Fujian Normal University

Chuangui Song ( $\sim$ Songcg1971@hotmail.com)

Department of Breast Surgery, Fujian Medical University Union Hospital No.29, Xin Quan Road, Gulou District, Fuzhou, Fujian Province, 350001, China

\section{Research article}

Keywords: TNBC, SUMO, prognostic, Chemotherapy, MYC

Posted Date: November 19th, 2020

DOl: https://doi.org/10.21203/rs.3.rs-109613/v1

License: (c) (1) This work is licensed under a Creative Commons Attribution 4.0 International License. Read Full License 


\section{Abstract}

Background: Triple negative breast cancer (TNBC) is a highly heterogeneous and aggressive disease. Developing new candidate biomarkers for chemotherapy response and possible therapeutic targets has become an urgent clinical need. Small ubiquitin-like modifier (SUMOs) mediate post-translational modifications (SUMOylation) that target multiple proteins was involved in many biological processes. The role of SUMOylation in TNBC remains to be elucidated.

Method: The expression of SUM01/2/3 were analyzed using TCGA and GEO databases $(\mathrm{N}=412)$. We also evaluated the SUM01/2/3 protein expression in 212 local TNBC patients using immunohistochemical $(\mathrm{IHC})$ staining. We then built a classifier with the LASSO Cox regression model based on associations between the expression of SUM01/2/3 proteins and the disease-free survival (DFS) of TNBC patients.

Results: Elevated SUM01/2/3 levels were indicated to be associated with a poorer overall survival (OS) and DFS for TNBC patients. Using the LASSO model, we built a classifier based on the IHC scores of SUM01/2/3 proteins and named it 'SB classifier'. Patients with the SB classifier defined high score were found to have an unfavorable response to chemotherapy (HR 4.04, 2.14-7.63; $p<0.0001)$. We then developed a nomogram to predict which patients might benefit from chemotherapy. Finally, our analysis provides insights on possible mechanisms that MYC activation causes the activation of SUMOylation.

Conclusion: We constructed a reliable prognostic and predictive tool for TNBC patients treated with chemotherapy. It may facilitate patient counseling and individualize management of TNBC patients. Meanwhile, our results suggested that the activation of SUMOylation pathway in TNBC may be induced by MYC signaling.

\section{Introduction}

Triple negative breast cancer (TNBC) is defined as a specific type of breast cancer by the absence of estrogen receptor (ER), progesterone receptor (PR), and human epidermal growth factor receptor-2 (HER2) expression. Although TNBC only constitutes to approximately $10-15 \%$ of all breast cancers ${ }^{[1,2]}$, it has a highly aggressive clinicopathological signatures and unfavorable outcomes ${ }^{[3]}$. Patients with TNBC generally develop distant metastasis within the first three years after initial treatment, with the mortality rate reaching about $40 \%$ in the first five years ${ }^{[4]}$. The lack of ER/PR and HER2 expression renders TNBC inaccessible to endocrine or anti-HER2 target therapies. Therefore, the most common treatment strategy for TNBC up to now is a combination of surgery, chemotherapy and radiotherapy. To date, anthracycline and taxane-based adjuvant chemotherapy is the standard regimen for TNBC patients after resection ${ }^{[5,6]}$. Triple negative breast cancer has a high sensitivity to chemotherapy, for patients with TNBC have an enhanced neoadjuvant response rate compared with other subtypes of breast cancer ${ }^{[7,8]}$. However, some of the patients still develop a rapid onset of recurrence and poor prognosis, which is commonly referred as "triple-negative paradox" [9]. Thus, identification of new predictive biomarkers for chemotherapy response and promising therapeutic targets might be beneficial in the treatment of TNBC. 
As an important post-translational protein modification, SUMOylation has attracted more and more attention. Four subtypes of SUMO have been identified including SUMO1, SUMO2, SUMO3 and SUMO4 ${ }^{[10]}$. SUMO2 and SUMO3 are $95 \%$ identical to each other and only $50 \%$ identical to SUMO1 ${ }^{[11]}$. SUM01/2/3 are ubiquitously expressed in human tissues, however SUMO4 is only expressed in spleen lymph nodes and kidney ${ }^{[12]}$. SUMOylation is catalyzed by a three-step enzymatic reaction, including activation, coupling and ligation ${ }^{[13]}$. SUMO E1-activating enzyme is a protein that contains two subunits, which are SUMO-activating enzyme E1 (SAE1) and SUMO-activating enzyme E2 (SAE2). UBC9 is the only known SUMO-conjugating E2 enzyme required for SUMOylation, and its deletion abolishes SUMO conjugation ${ }^{[14]}$. SUMO E3 ligases are roughly divided into three categories including the protein inhibitor of activated STAT-1 (PIAS) protein family, the nucleoporin Ran binding protein 2 and the human polycomb protein Pc2. Although SUMO is similar to ubiquitin, SUMOylation does not directly lead to protein degradation, but leads to the regulation of cell functions, such as protein-protein interactions, maintaining genome integrity, subcellular localization, transcription regulation, DNA repair and cell cycle ${ }^{[11,15]}$. The dysregulation of SUMOylation could result in tumors progression and is considered as new novel biomarkers and possible therapeutic targets for cancers ${ }^{[16]}$.

In this study, we sought to explore the expression and prognostic utility of SUMOs and aimed to build a prognosis prediction model based on SUMO1/2/3 protein expression. Meanwhile, potential mechanisms that regulate SUMOylation pathway in TNBC were also explored.

\section{Material And Methods}

\section{Extraction of gene expression data from TNBC patient datasets}

The microarray datasets of TNBC patients were extracted from Gene Expression Omnibus (GEO) database (http://www.ncbi.nlm.nih.gov/geo/) and TCGA database (http://www.cbiop ortal.org/data_sets.jsp). Four microarray gene expression datasets of TNBC patient, GSE31448, GSE45827, GSE53752 and GSE65216 were obtained from GEO database. The method for extracting microarray gene expression values is based on our previous research ${ }^{[17-19]}$.

\section{Patients and specimens}

A total of 212 TNBC patients were retrospectively reviewed from Fujian Medical University Union Hospital between June 2013 and August 2017. All patients were histologically confirmed TNBC, ranging from 27 to 77 years of age (median: 51years) with 4-77 months of follow-up information. The clinicopathological data included age, tumor size, nodal status, tumor grade, lymphovascular invasion, type of surgery, chemotherapy and radiotherapy information were obtained from medical records. Disease-free survival (DFS) was defined as the time of diagnosis to the date of clinical relapse (with histopathology confirmation or radiological evidence of tumor recurrence). Overall survival (OS) was defined as the time of diagnosis until death from any cause. The follow-up deadline was August 30, 2020. 
The standard requirements for patients included in the study were: (a) no history of other malignancy tumor, not bilateral breast cancer, not de novo IV stage; (b) received total mastectomy or breast conserving surgery without neoadjuvant chemotherapy or radiotherapy; (c) primary tumor size was pT1cpT2; (d) demographic, clinicopathological and follow-up information were complete. Patients who received at least 3 cycles of anthracycline-based and 3 cycles of taxanes-based regimens (4EC-4T, 3FEC3T, 6TEC) were considered as having chemotherapy, while those with insufficient cycles of chemotherapy were excluded from this study.

This study was approved by the Research Ethics Committee of Fujian Medical University Union Hospital. Informed consent was obtained from each participant.The patient's information was shown in Supplementary Table 1.

\section{Immunohistochemistry (IHC) staining analysis}

IHC staining analysis was performed to measure the SUM01/2/3 protein expression in all TNBC tissues and adjacent normal breast tissues according to standard immunoperoxidase staining procedure. Slides were incubated with anti-SUMO1 (ab32058, abcam, diluted 1:150), anti-SUMO2 (ab233222, abcam, diluted 1:300) and anti-SUMO3 (ab203570, abcam, diluted 1:300) according to the manufacturer's instructions. To ensure quality, a negative control was prepared by the substitution of primary antibody with phosphate-buffered saline (PBS, $5 \%$ BSA). The IHC staining scores of SUM01/2/3 were assessed by two independent pathologists. The percentage of stained positive cells was scored from 1 to 4: 1, 0-25\%; $2,26-50 \% ; 3,51-75 \%$ and $4,75-100 \%$. The staining intensity score was calculated from 0 to $3: 0$, no staining; 1 , weak staining; 2 , moderate staining; and 3 , strong staining. The final scores were based on the sum of these two scores. A score more that 5 was defined as high expression level and a score less than and include 5 wan defined as low expression.

\section{Gene set variation analysis (GSVA) and LASSO analysis}

GSVA provides increased power to detect subtle pathway activity changes over a sample population in comparison to corresponding methods. In the research, the pathway activity of protein SUMOylation and 50 oncogene pathways in TNBC were analyzed. The GSVA analysis was performed using R package 'GSVA'. We used the LASSO Cox regression model to constructed a 3 SUMOs-based classifier (SB classifier) for predicting the DFS of TNBC patients. The LASSO analysis was performed using R package 'glmnet'.

\section{Statistical analysis}

In the research, $t$ test was used to compared continuous variables in two groups by using GraphPad Prism 5.0 software. Correlations between SUM01/2/3 expression and clinicopathological characteristics were conducted by the chi-squared test. DFS and OS were calculated by the Kaplan-Meier method by using SPSS, and differences between groups were tested by log-rank test. We performed cox regression 
analysis to undertake the univariate and multivariate survival analysis. All $p$ values $<0.05$ were considered statistically significant.

\section{Results}

\section{Protein SUMOlyation pathway was activated in TNBC}

In order to explore the pathway activity of protein SUMOlyation in TNBC, TCGA database and four related GEO databases (GSE31448, GSE45827, GSE53752 and GSE65216) were employed. TCGA database contained 166 cases of TNBC tissues and 113 adjacent normal breast tissues. For the remaining four GEO databases, 98 cases of TNBC tissues and 31 adjacent normal breast tissues were from GSE31448 database, 41 cases of TNBC tissues and 11 adjacent normal breast tissues were from GSE45827 database, GSE53752 database consisted of 51 cases of TNBC tissues and 25 adjacent normal breast tissues, while 55 cases of TNBC tissues and 10 adjacent normal breast tissues were retrieved in GSE65216 database. GSVA was performed to conduct GO analysis and assign protein SUMOlyation pathway activity estimates to individual samples from TCGA and GEO databases. It is indicated that protein SUMOlyation pathway exhibited a higher enrichment score in the TNBC tissues compared with adjacent normal breast tissues (GSE45827, GSE65216, $p<0.001$; GSE31448, GSE53752, $p<0.01$; TCGA, $p<0.05$ ) (Figure 1A-E). Moreover, meta-analysis containing 603 tissues from 5 TNBC databases mentioned above further demonstrated that protein SUMOlyation pathway was activated in TNBC $(p<0.001$; Figure 1F).

\section{High SUM01/2/3 protein expression were unfavorable prognostic for TNBC patients}

To investigate whether SUM01, SUMO2 and SUMO3 were involved in TNBC progressionwe analyzed SUMO1, SUMO2 and SUMO3 expression in the TCGA and four GEO databases. SUMO1, SUMO2 and SUMO3 were up-regulated in TNBC tissues compared with adjacent normal breast tissues in TCGA, GSE31448, GSE45827 and GSE65216 (Figure 2A, B, C\&E). In GSE53752, up-regulation of SUMO3 expression and down-regulation of SUMO1 and SUMO2 in TNBC tissues compared with adjacent normal breast tissues were observed (SUM01, $p<0.01$; SUMO2, $p>0.05$; SUM03, $p<0.001$ ) (Figure 2D). Remarkably, meta-analysis revealed that the mRNA expression of SUMO1, SUMO2 and SUMO3 were increased in TNBC (Figure 2F-H).

In order to further validate TCGA and GEO expression data, we performed an IHC study using local patient samples to determine the protein expression of SUMO1, SUMO2 and SUMO3 in TNBC. The IHC analysis showed that SUMO1, SUMO2 and SUMO3 were significantly upregulated in 212 TNBC tissues compared with paired adjacent normal breast tissues (Figure 3). The clinicopathological characteristics of these patients in the study cohort are summarized in Table 1. Most of the patients (93.4\%) were treated with adjuvant chemotherapy. We estimated the correlations of SUM01/2/3 expression with relevant clinicopathological factors. No associations between SUMO1 expression and clinicopathological features were observed. SUMO2 expression were indicated to be significantly associated with tumor size 
$(p=0.032)$, while SUMO3 expression correlated significantly with the lymph node metastasis $(p=0.033)$ and lymphovascular invasion $(p=0.028)$.

Survival analysis was conducted to explore the relationship between SUM01/2/3 proteins expression, clinicopathological factors along with survival of these 212 TNBC patients. Kaplan-Meier survival analysis of OS and DFS of TNBC patients was generated according to SUM01/2/3 protein expression (Figure 4A-F), which implied that TNBC patients with higher expression of SUM01/2/3 suffer lower possibility of OS (Figure 4A-C) and DFS (Figure 4D-F). Univariate and multivariate Cox regression analyses were employed to clarify the independent factors affecting OS and DFS of TNBC patients.

In order to see which one is an independent factor impacting patient outcome, univariate analysis and multivariate Cox analyses were performed. Lymph node metastasis, radiotherapy, SUMO1, SUMO2 and SUMO3 protein expression were finally determined to be independent prognostic factors for OS of TNBC patients by multivariate Cox analyses (Figure 4G). As for DFS, tumor size, lymph node metastasis, tumor grade, lymphovascular invasion and $\mathrm{SUMO} 3$ protein expression were determined to be independent prognostic factors in TNBC patients (Figure 4G).

\section{Construction of a prognostic scoring model based on SUM01/2/3 proteins}

In order to construct a risk score model for predicting DFS of TNBC, we used LASSO Cox regression model to build a SUMO proteins-based prognostic classifier, which included SUMO1, SUMO2 and SUMO3 and named it SB classifier (Figure 5A\&B). Using the LASSO Cox regression models, we calculated a risk score for each patient based on individualized values of $\mathrm{IHC}$ scores for the three proteins: Risk score $=$ $($ SUM01×0.3746) $+($ SUMO2×0.3290 $)+($ SUMO3×0.8217). The SB classifier showed significantly higher prognostic accuracy than single SUMO alone (Figure $5 \mathrm{C}$ ). When we assessed the distribution of risk scores and recurrence status, TNBC patients with higher risk scores generally had higher recurrence rate than those with lower risk scores (Figure 5D). TNBC patients were then assigned into a SB classifier highlevel group (75 patients) and low-level group (137 patients) by the cut-off value (5.87). The Kaplan-Meier curve showed the patients in the SB classifier high-level group presented a significantly worse DFS (HR $2.8,95 \% \mathrm{Cl} 1.73-4.53, p<0.01$ ) (Figure $5 \mathrm{E}$ ). By predicting DFS of TNBC patients at 1,3 , and 5 years, the areas under the curve (AUC) of the ROC curves obtained from the risk-based prediction model were 0.84 , 0.7 and 0.7 (Figure 5F). Total cohort was randomly divided into two equal training and validation sets by using X-tile plots. Based on cut-points of risk score, TNBC patients were divided into SB classifier lowlevel and SB classifier high-level in the training cohort, patients with poor DFS displayed higher risk score than those with good prognosis (Figure 5G). Similar prognostic results were found in validation cohort and total cohort (Figure 5H\&l).

Results from survival analysis by our SB classifier showed that patients in the classifier-defined low score group had a favourable response to chemotherapy (HR=4.04, 2.14-7.63; $p<0.0001)$ (Figure 6A), which indicated that our SB classifier could successfully identify patients with TNBC who might benefit from chemotherapy. To provide the clinician with a quantitative method to predict probability of cancer 
recurrence for TNBC patients with chemotherapy, we constructed a nomogram that integrated both the SB classifier and clinicopathological factors (figure 6B).

\section{Oncogenic pathways that positive correlate to protein SUMOylation were activated in the tumors of TNBC patients}

By using GSVA method and the Molecular Signatures Database hallmark gene set collection, we analyzed the mRNA expression data of TNBC in TCGA, GSE53752, GSE65216 and GSE31448 database. The correlation between protein SUMOylation and 50 hallmark gene set in TNBC was analyzed by pearson correlation analysis. In the tumor samples of the TNBC cohort, the intersection of TCGA, GSE53752, GSE65216 and GSE31448 datasets revealed that there was a positive correlation existed between protein SUMOylation and E2F-targets, MYC-targets-V1, Mtorc1-signaling, Mitotic-spindle, G2Mcheckpoint and unfolded protein response ( $r>0.3$, Figure $7 A-E)$. In addition, there was a positive correlation existed between protein SUMOylation and Mitotic-spindle, G2M-checkpoint and Unfolded protein response in the intersection of TCGA, GSE53752, GSE65216 and GSE31448 normal tissues datasets $(r>0.3$, Figure 7F-J). The intersection of these two arrays was shown in Figure 7K, and 3 overlapping pathway including Mitotic-spindle, G2M-checkpoint and unfolded protein response were found. Next, we analyzed the pathway activity of E2F-targets, MYC-targets-V1, Mtorc1-signaling, Mitotic-spindle, G2Mcheckpoint and unfolded protein response in the TCGA and GEO databases. These six pathways were upregulated in TNBC tissues compared with adjacent normal breast tissues in TCGA, GSE53752, GSE65216 and GSE31448 (Figure 8A-D). Finally, meta-analysis revealed that the pathway activity of E2F-targets, MYC-targets-V1, Mtorc1-signaling, Mitotic-spindle, G2M-checkpoint and unfolded protein response were increased in TNBC (Figure 8E-J).

\section{Discussion}

TNBC was characterized by high invasiveness and has a worse prognosis than other subtypes of breast cancer. For the lack of ER, PR and HER2 expression, there is no specific systemic treatment such as endocrine therapy or anti-HER2 targeted therapy. Currently, the basis of TNBC treatment is surgery, chemotherapy and radiotherapy. Anthracycline and taxane-based chemotherapy regimen is the standard

of care for prevention of TNBC recurrence and survival improvement ${ }^{[5]}$. EBCTCG analysis demonstrated a moderate reduction in 5-year and 10-year risk of recurrence and death with a dose intensity adjuvant chemotherapy, especially for TNBC patients ${ }^{[6]}$. However, some of the patients still develop a rapid onset of recurrence and poor prognosis after conventional chemotherapy. Thus, identification of novel biomarkers which could be used to predict chemotherapy response and promising therapeutic targets might be beneficial in the treatment of TNBC.

Previous studies indicated that SUMOylation is closely related to carcinogenesis, tumor proliferation and metastasis, and significantly up-regulated in most cancers ${ }^{[20-23]}$. Therefore, SUMOylation may become a potential target for cancer treatment. However, the expression and underlying mechanisms of SUMOylation remain poorly understood in TNBC. In the present research, we advanced the knowledge of 
the role of SUMOylation in TNBC. We demonstrated that the pathway activity of protein SUMOlyation and the expression of SUM01/2/3 mRNA were increased in TNBC tissues compared with adjacent normal breast tissues by TCGA and GEO database. Meanwhile, our immunohistochemistry staining results suggested that the expression of SUM01/2/3 proteins were significantly increased in tumor tissues of 211 TNBC patients. According to survival analysis, SUM01/2/3 protein expression levels were all associated with disease free survival and overall survival of TNBC patients. In addition, we developed a novel prognostic tool based on IHC scores of SUM01/2/3 to improve the prediction of disease recurrence for TNBC patients. Further use of SB classifier might allow for better identification of TNBC patients who are most likely to benefit from chemotherapy. Therefore, the classifier for TNBC patients is both a prognostic and predictive method. Patients with a SB classifier defined low score might have both a lower likelihood of recurrence and a clear benefit from chemotherapy.

Meanwhile, we analyzed the pathways associated with SUMOylation in TNBC. Our data showed that E2Ftargets, MYC-targets-V1, Mtorc1-signaling, Mitotic-spindle, G2M-checkpoint and unfolded protein response were positively correlated with SUMOylation in tumor tissues of TNBC patients. However, only Mitotic-spindle, G2M-checkpoint and unfolded protein response were found positively correlated with SUMOylation in normal tissues of TNBC patients. MYC is an important transcription factor. MYC mutations lead to uncontrolled expression of many genes, some of which are involved in cell proliferation and relate to the development of cancer. MYC protein activates the transcription of SUMO activating enzyme subunit 1 (SAE1) by directly binding to the classic E-Box sequence located near the SAE1 transcription start site ${ }^{[24]}$. Inhibition of SUMOylation was reported to disable MYC-induced cell proliferation and triggers $\mathrm{G} 2 / \mathrm{M}$ cell cycle arrest in mouse and human MYC-driven lymphomas ${ }^{[25]}$. In addition, there is accumulating evidence that SUMO directly and indirectly regulated protein localization within the mitotic spindle. AMP-activated protein kinase (AMPK) inhibits protein synthesis through suppression of mammalian target of rapamycin complex 1 (mTORC1). SUMOylation of AMPKa1 attenuates AMPK activation, and then prompts restoration of mTORC1 signaling ${ }^{[26]}$. Retinoblastoma protein $(\mathrm{Rb})$ is a prototypical tumor suppressor Hypo-phosphorylated $\mathrm{Rb}$ is related to G0/G1 arrest by inhibiting the activity of E2F transcription factors, while hyper-phosphorylation Rb releases $\mathrm{E} 2 \mathrm{~F}$ and makes the cell cycle from $\mathrm{G0} / \mathrm{G} 1$ into $S$ phase. SUMOylation of Rb causes Rb hyperphosphorylation and E2F-1 release ${ }^{[27]}$. X-boxbindingprotein 1 (XBP1) is a key transcription factor that regulates the endoplasmic reticulum (ER) stress response, which is a cytoprotective mechanism that deals with the accumulation of unfolded protein in the ER. When endoplasmic reticulum stress occurs, unspliced XBP1 mRNA is converted into mature mRNA, and the transcription factor pXBP1 is translated and the transcription of endoplasmic reticulum related genes is activated to process unfolded proteins ${ }^{[28]}$. SUMO-conjugase, UBC9, specifically bound to the leucine zipper motif of pXBP1 and increased the stability of pXBP1. Our analysis provides insights on possible mechanism that MYC activation causes the activation of SUMOylation, which eventually results in the activation of E2F-targets, Mtorc1-signaling, Mitotic-spindle, G2M-checkpoint and unfolded protein response. 
The major strengths of the present study are that it had a large enough sample size of TNBC patients to perform survival analysis based on SUM01/2/3 proteins, and developed a prognostic nomogram. In addition, some small molecule drugs that inhibit SUMOylation have been considered for the treatment of cancer. SUMO E1 inhibitor ML-792 is currently being tested in a phase 1 clinical trial for patients with metastatic solid tumors and lymphomas. In the current era of precision medicine, using the prognostic biomarker to select eligible patients and administration of specific treatments is a promising strategy. Our findings suggested that the inhibition of SUMOylation could be a promising therapeutic strategy for the treatment of TNBC patients.

Undoubtedly, there are several limitations in this study. Firstly, as all TNBC patients are Chinese and from one single center. The findings of the present study may not be generalizable to all populations. Secondly, more intensive researches are still warranted to illustrate the underlying mechanisms in regulation of SUMOylation for TNBC.

\section{Conclusion}

In summary, our finding showed that pathway activity of SUMOylation, SUM01/2/3 mRNA and protein levels were up-regulated in TNBC patients based on TCGA, GEO and 212 TNBC specimens. Three SUMOsbased prognostic model can effectively classify TNBC patients into groups at low and high risk of disease recurrence. Moreover, our study showed that the SB classifier might be a useful predictive tool for TNBC patient treated with chemotherapy (Figure 9). Thus, the SB classifier potentially offers clinical value in directing personalized therapeutic regimen selection for TNBC patients. Meanwhile, our analysis provides insights on possible mechanisms that MYC activation leads to the activation of SUMOylation, which eventually causes the activation of E2F-targets, Mtorc1-signaling, Mitotic-spindle, G2M-checkpoint and unfolded protein response.

\section{Abbreviations}

TNBC: Triple negative breast cancer

SUMOs: Small ubiquitin-like modifier

IHC: Immunohistochemical

DFS: Disease-free survival

OS: Overall survival

ER: Estrogen receptor

PR: Progesterone receptor

HER2: Human epidermal growth factor receptor-2 
SAE1『SUMO-activating enzyme E1

SAE2 \SUMO-activating enzyme E2

PIAS $\triangle$ Protein inhibitor of activated STAT-1

AUC $₫$ Areas under the curve

\section{Declarations}

\section{Availability of data and materials}

The datasets used and/or analyzed during the current study are available from the corresponding author on reasonable request.

\section{Acknowledgements}

Not applicable

\section{Funding}

This research was funded by National Natural Science Foundation of China (81672817), joint funds for the Innovation of Science and Technology, Fujian Province (2019Y9054), the fund of Natural Science Foundation of Fujian Province (2020J01311402), National Science Foundation for Young Scientists of China (82003095) and Fujian Provincial Key Laboratory of Hepatic Drug Research (KFLX2020001).

\section{Author information}

\section{Affiliations}

Yuxiang Lin1,2,3, Qingshui Wang4,5,6, Han Xiao7, Zhiwei Chen8, Meichen Jiang7, Jie Zhang1,2,3, Rongrong Guo1,2,3, Shaohong Kang1,2,3, Yao Lin4,5 and Chuangui Song1,2,3

Department of Breast Surgery, Fujian Medical University Union Hospital, Fuzhou, Fujian Province, 350001, China

Yuxiang Lin, Jie Zhang,Rongrong Guo, Shaohong Kang and Chuangui Song

Department of General Surgery, Fujian Medical University Union Hospital, Fuzhou, Fujian Province, 350001, China

Yuxiang Lin, Jie Zhang,Rongrong Guo, Shaohong Kang and Chuangui Song

\section{Breast Cancer Institute, Fujian Medical University, Fuzhou, Fujian Province, 350001, China}

Yuxiang Lin, Jie Zhang,Rongrong Guo, Shaohong Kang and Chuangui Song 
Central Laboratory at The Second Affiliated Hospital of Fujian Traditional Chinese Medical University, Collaborative Innovation Center for Rehabilitation Technology, Fujian University of Traditional Chinese Medicine, Fuzhou, Fujian Province, 350001, China.

Qingshui Wang and Yao Lin

Key Laboratory of Optoelectronic Science and Technology for Medicine of Ministry of Education, College of Life Sciences, Fujian Normal University, Fuzhou, Fujian Province, 350001, China

Qingshui Wang and Yao Lin

Fujian Provincial Key Laboratory of Hepatic Drug Research, Fuzhou, Fujian Province, 350001, China Qingshui Wang

Department of Pathology, Fujian Medical University Union Hospital, Fuzhou, Fujian Province, 350001, China

Han Xiao and Meichen Jiang

Department of Gynecology and Obstetrics, Fujian Maternity and Child Health Hospital, Affiliated Hospital of Fujian Medical University, Fuzhou, Fujian Province, 350001, China

Zhiwei Chen

Authors' contributions

Y L and CG S conceptualized and designed the study. YX L, QS W, and $H X$ drafted and wrote the manuscript. ZW C and MC J performed the immunohistochemistry and analyzed the results. J Z, and RR $\mathrm{G}$ collected and organized the clinical samples and data. SH K performed the statistical analysis on the clinical data. All authors contributed to the data interpretation and reviewed and approved the final version of the manuscript.

Corresponding author

Correspondence to Yao Lin and ChuanGui Song.

Ethics declarations

Ethics approval and consent to participate

This study was approved by the Research Ethics Committee of Fujian Medical University Union Hospital. Informed consent was obtained from each participant.

Consent for publication 
Not applicable

Competing interests

The authors declare that they have no competing interests.

\section{References}

1. Dent R, Trudeau M, Pritchard KI, Hanna WM, Kahn HK, Sawka CA, et al. Triple-negative breast cancer: clinical features and patterns of recurrence. Clinical cancer research : an official journal of the American Association for Cancer Research 2007; $13(15$ Pt 1):4429-4434.

2. Burstein MD, Tsimelzon A, Poage GM, Covington KR, Contreras A, Fuqua SA, et al. Comprehensive genomic analysis identifies novel subtypes and targets of triple-negative breast cancer. Clinical cancer research : an official journal of the American Association for Cancer Research 2015; 21(7):1688-1698.

3. Abramson VG, Lehmann BD, Ballinger TJ, Pietenpol JA. Subtyping of triple-negative breast cancer: implications for therapy. Cancer 2015; 121(1):8-16.

4. Denkert C, Liedtke C, Tutt A, von Minckwitz G. Molecular alterations in triple-negative breast cancerthe road to new treatment strategies. Lancet 2017; 389(10087):2430-2442.

5. Budd GT, Barlow WE, Moore HC, Hobday TJ, Stewart JA, Isaacs C, et al. SWOG S0221: a phase III trial comparing chemotherapy schedules in high-risk early-stage breast cancer. Journal of clinical oncology : official journal of the American Society of Clinical Oncology 2015; 33(1):58-64.

6 . Increasing the dose intensity of chemotherapy by more frequent administration or sequential scheduling: a patient-level meta-analysis of $\mathbf{3 7} \mathbf{2 9 8}$ women with early breast cancer in 26 randomised trials. Lancet 2019; 393(10179):1440-1452.

7. Cortazar P, Zhang L, Untch M, Mehta K, Costantino JP, Wolmark N, et al. Pathological complete response and long-term clinical benefit in breast cancer: the CTNeoBC pooled analysis. Lancet 2014; 384(9938):164-172.

8. Liedtke C, Mazouni C, Hess KR, André F, Tordai A, Mejia JA, et al. Response to neoadjuvant therapy and long-term survival in patients with triple-negative breast cancer. Journal of clinical oncology : official journal of the American Society of Clinical Oncology 2008; 26(8):1275-1281.

9. Fornier M, Fumoleau P. The paradox of triple negative breast cancer: novel approaches to treatment. The breast journal 2012; 18(1):41-51.

10. Wang Q, Zhang X, Chen L, Weng S, Xia Y, Ye Y, et al. Regulation of the Expression of DAPK1 by SUMO Pathway. Biomolecules 2019; 9(4).

11. Satpathy S, Guérillon C, Kim TS, Bigot N, Thakur S, Bonni S, et al. SUMOylation of the ING1b tumor suppressor regulates gene transcription. Carcinogenesis 2014; 35(10):2214-2223.

12. Dalmasso G, Nguyen HTT, Faïs T, Massier S, Barnich N, Delmas J, et al. Crohn's Disease-Associated Adherent-Invasive Escherichia coli Manipulate Host Autophagy by Impairing SUMOylation. Cells 
2019; 8(1).

13. Gareau JR, Lima CD. The SUMO pathway: emerging mechanisms that shape specificity, conjugation and recognition. Nature reviews Molecular cell biology 2010; 11(12):861-871.

14. Wang QE, Zhu Q, Wani G, El-Mahdy MA, Li J, Wani AA. DNA repair factor XPC is modified by SUMO-1 and ubiquitin following UV irradiation. Nucleic Acids Res 2005; 33(13):4023-4034.

15. Becker J, Barysch SV, Karaca S, Dittner C, Hsiao HH, Berriel Diaz M, et al. Detecting endogenous SUMO targets in mammalian cells and tissues. Nat Struct Mol Biol 2013; 20(4):525-531.

16. Boulanger M, Paolillo R, Piechaczyk M, Bossis G. The SUMO Pathway in Hematomalignancies and Their Response to Therapies. Int J Mol Sci 2019; 20(16).

17. Wang Q, Ye Y, Lin R, Weng S, Cai F, Zou M, et al. Analysis of the expression, function, prognosis and co-expression genes of DDX20 in gastric cancer. Computational and structural biotechnology journal 2020; 18:2453-2462.

18. Wang QS, Li F, Liao ZQ, Li K, Yang XL, Lin YY, et al. Low level of Cyclin-D1 correlates with worse prognosis of clear cell renal cell carcinoma patients. Cancer Med 2019; 8(9):4100-4109.

19. Li F, Wang Q, Xiong X, Wang C, Liu X, Liao Z, et al. Expression of 4E-BP1 and phospho-4E-BP1 correlates with the prognosis of patients with clear cell renal carcinoma. Cancer Manag Res 2018; 10:1553-1563.

20. Hendriks IA, Vertegaal AC. A comprehensive compilation of SUMO proteomics. Nature reviews Molecular cell biology 2016; 17(9):581-595.

21. Bogachek MV, Park JM, De Andrade JP, Lorenzen AW, Kulak MV, White JR, et al. Inhibiting the SUMO Pathway Represses the Cancer Stem Cell Population in Breast and Colorectal Carcinomas. Stem cell reports 2016; 7(6):1140-1151.

22. He X, Riceberg J, Soucy T, Koenig E, Minissale J, Gallery M, et al. Probing the roles of SUMOylation in cancer cell biology by using a selective SAE inhibitor. Nature chemical biology 2017; 13(11):11641171.

23. Li R, Wei J, Jiang C, Liu D, Deng L, Zhang K, et al. Akt SUMOylation regulates cell proliferation and tumorigenesis. Cancer research 2013; 73(18):5742-5753.

24. Amente S, Lavadera ML, Palo GD, Majello B. SUMO-activating SAE1 transcription is positively regulated by Myc. American journal of cancer research 2012; 2(3):330-334.

25. Hoellein A, Fallahi M, Schoeffmann S, Steidle S, Schaub FX, Rudelius M, et al. Myc-induced SUMOylation is a therapeutic vulnerability for B-cell lymphoma. Blood 2014; 124(13):2081-2090.

26. Yan Y, Ollila S, Wong IPL, Vallenius T, Palvimo JJ, Vaahtomeri K, et al. SUMOylation of AMPKa1 by PIAS4 specifically regulates mTORC1 signalling. Nat Commun 2015; 6:8979.

27. Meng F, Qian J, Yue H, Li X, Xue K. SUMOylation of Rb enhances its binding with CDK2 and phosphorylation at early G1 phase. Cell cycle (Georgetown, Tex) 2016; 15(13):1724-1732.

28. Uemura A, Taniguchi M, Matsuo Y, Oku M, Wakabayashi S, Yoshida H. UBC9 regulates the stability of XBP1, a key transcription factor controlling the ER stress response. Cell structure and function 2013; 
38(1):67-79.

\section{Tables}

Due to technical limitations, table 1 is only available as a download in the Supplemental Files section.

\section{Figures}
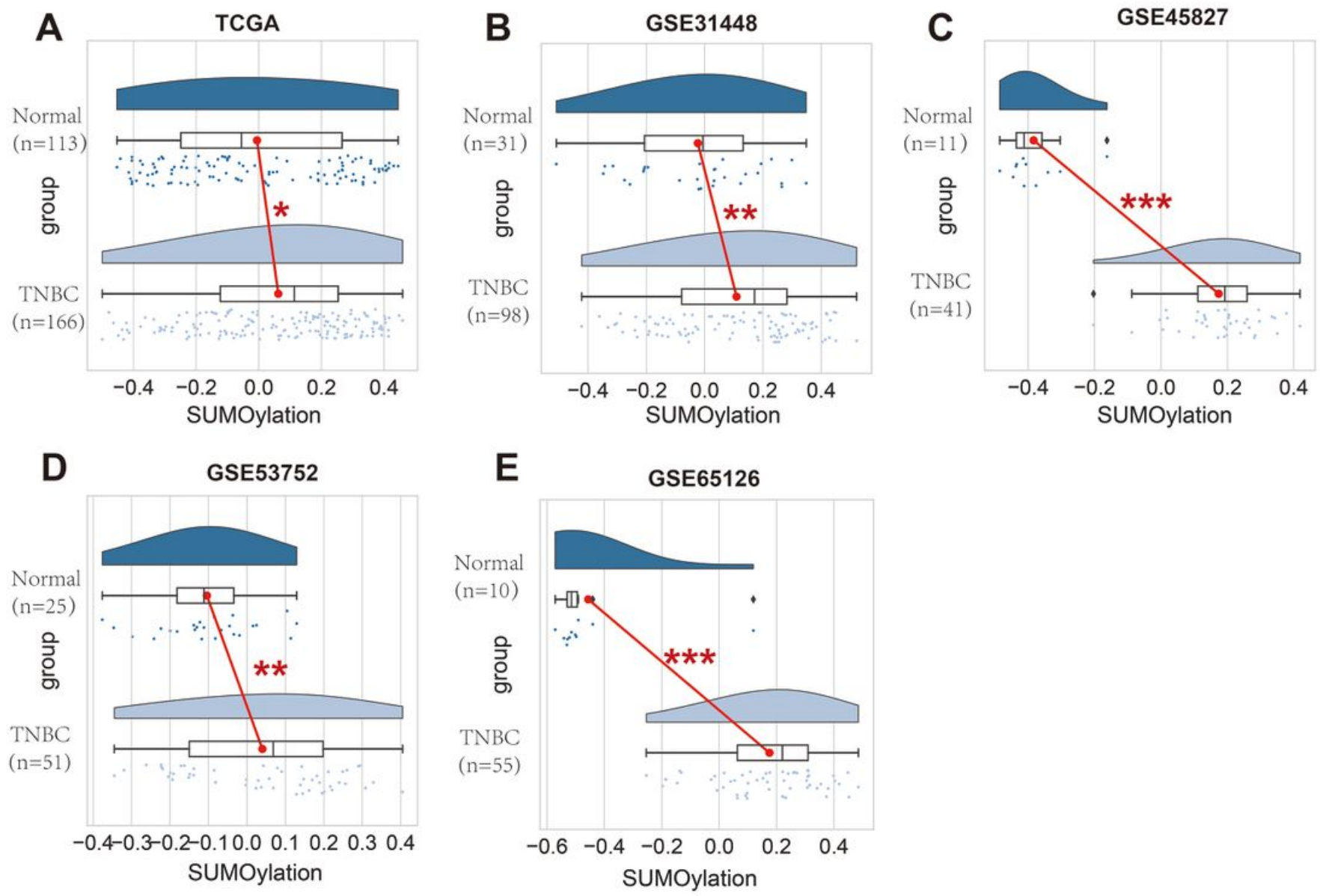

$\mathbf{F}$

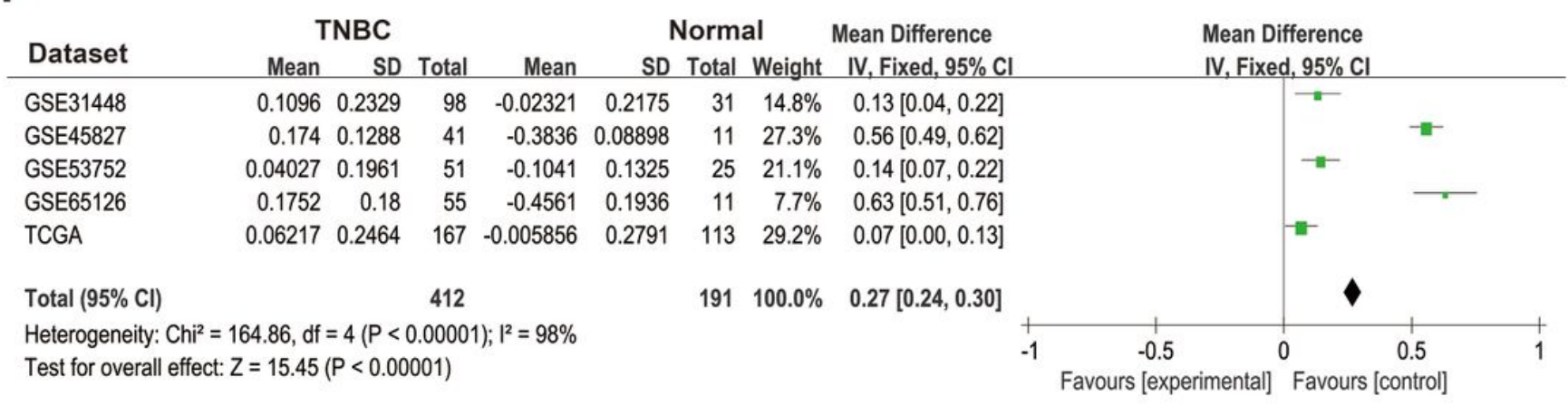

\section{Figure 1}

The pathway activity of SUMOylation is up-regulated in TNBC. (A-E) The pathway activity of SUMOylation in TNBC tumor tissues and normal breast tissue of TCGA (A), GSE31448 (B), GSE45827 (C), GSE53752 
(D) and GSE65216 (E) based on GSVA analysis. (F) Meta-analysis of pathway activity of SUMOylation in TNBC patients based on TCGA, GSE31448, GSE45827, GSE53752 and GSE65216. *, p<0.05; **, p<0.01; $\star \star *, p<0.001$.
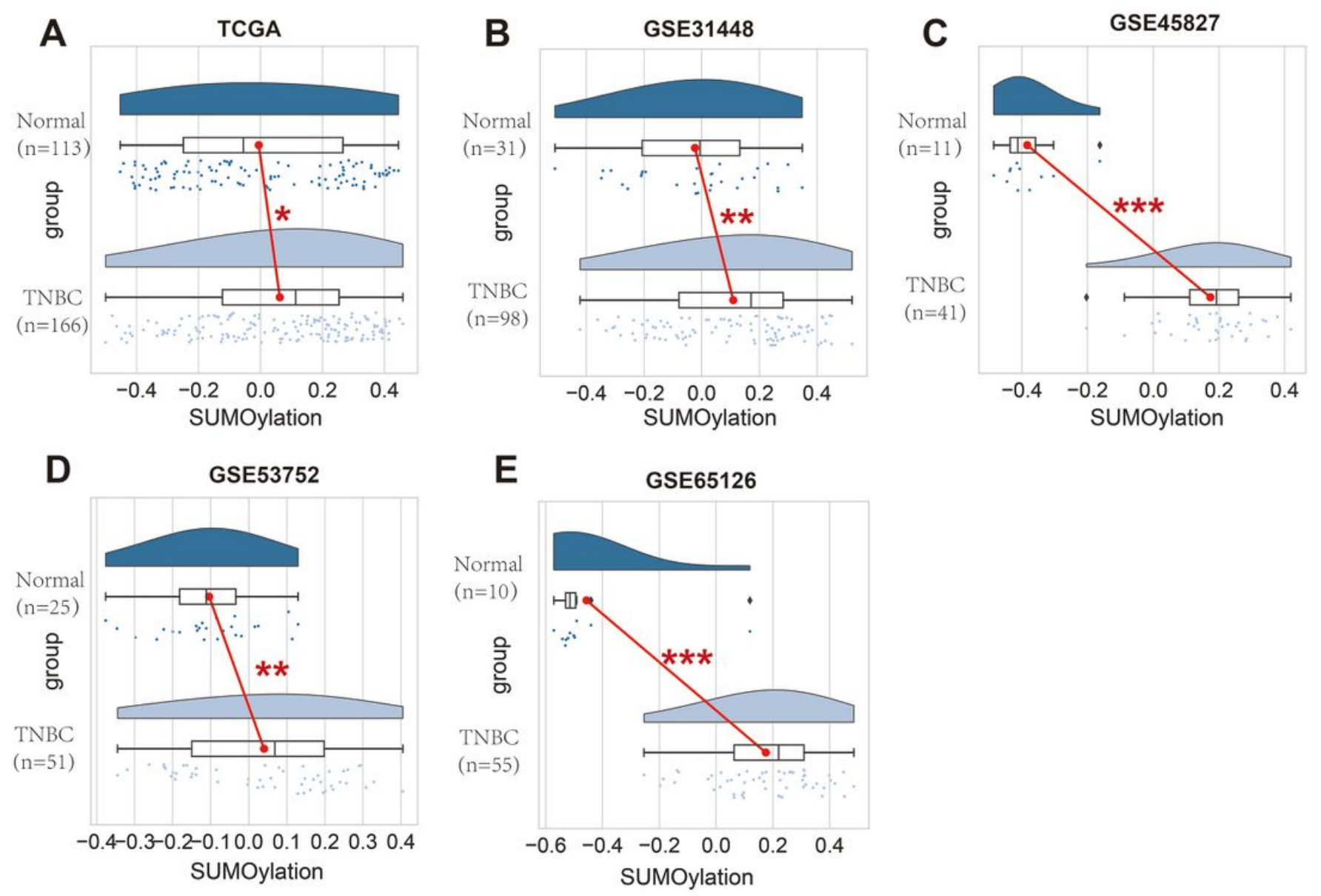

$\mathbf{F}$

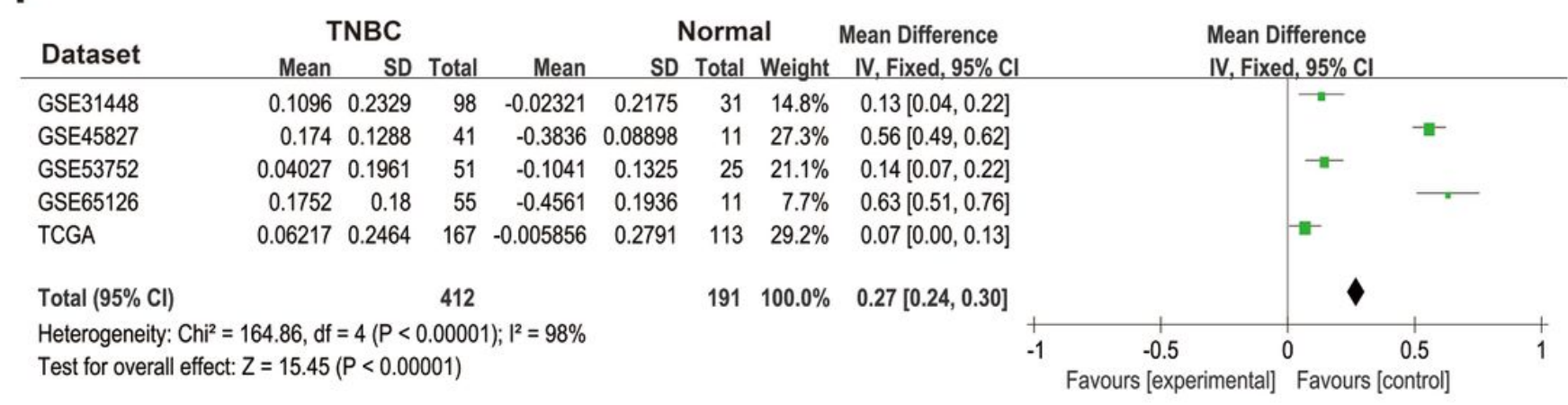

\section{Figure 1}

The pathway activity of SUMOylation is up-regulated in TNBC. (A-E) The pathway activity of SUMOylation in TNBC tumor tissues and normal breast tissue of TCGA (A), GSE31448 (B), GSE45827 (C), GSE53752 (D) and GSE65216 (E) based on GSVA analysis. (F) Meta-analysis of pathway activity of SUMOylation in TNBC patients based on TCGA, GSE31448, GSE45827, GSE53752 and GSE65216. *, $p<0.05 ; * \star, p<0.01$; $\star \star \star, ~ p<0.001$. 

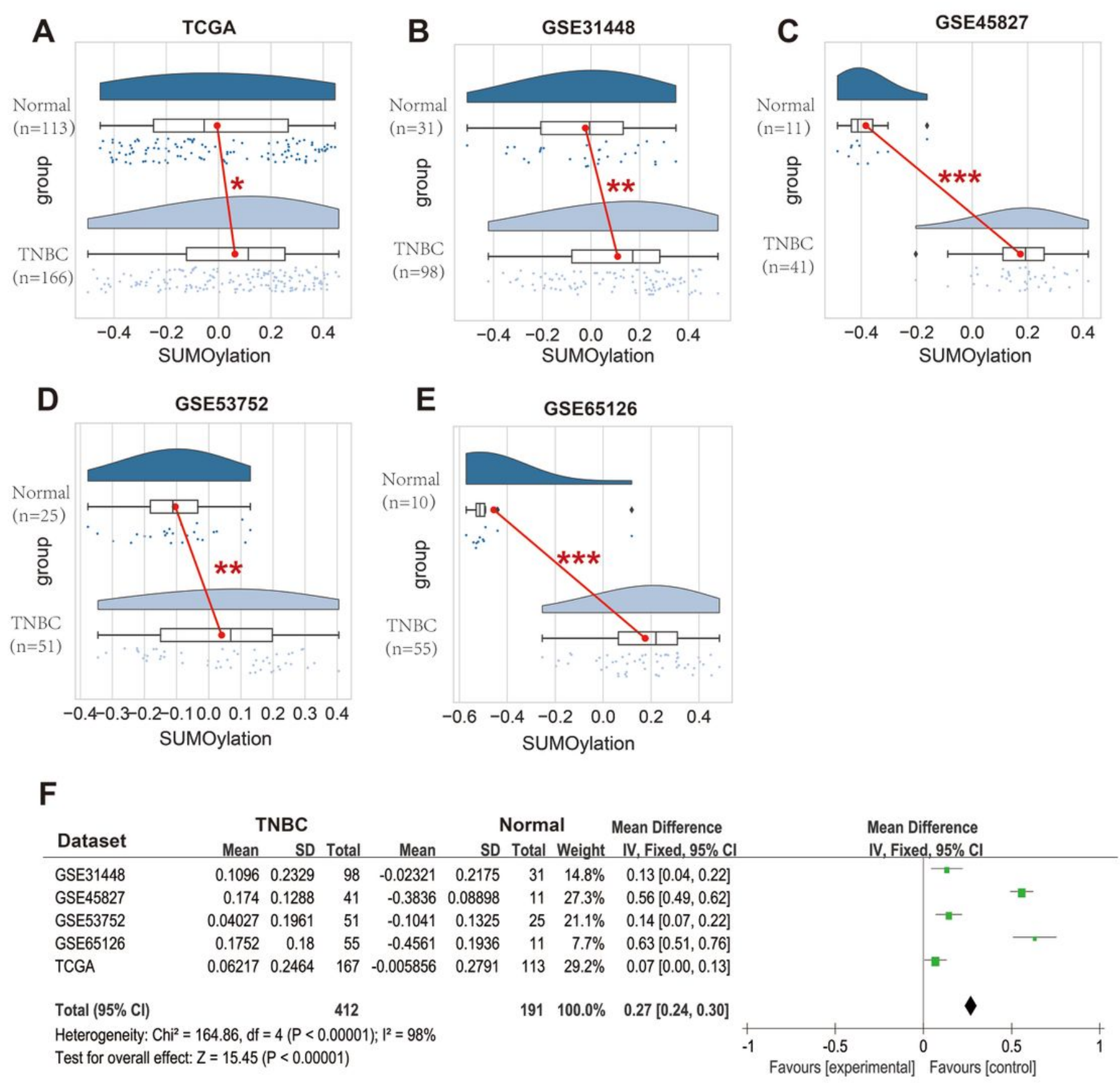

\section{Figure 1}

The pathway activity of SUMOylation is up-regulated in TNBC. (A-E) The pathway activity of SUMOylation in TNBC tumor tissues and normal breast tissue of TCGA (A), GSE31448 (B), GSE45827 (C), GSE53752 (D) and GSE65216 (E) based on GSVA analysis. (F) Meta-analysis of pathway activity of SUMOylation in TNBC patients based on TCGA, GSE31448, GSE45827, GSE53752 and GSE65216. *, $p<0.05 ;$ **, $p<0.01$; $\star \star \star, p<0.001$. 


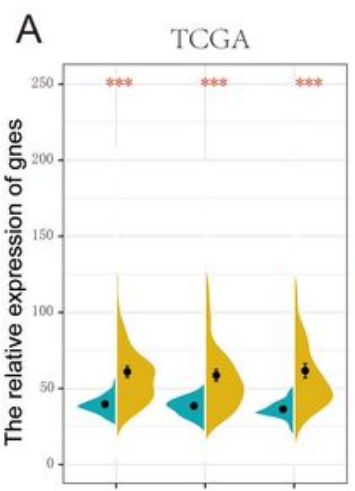

D

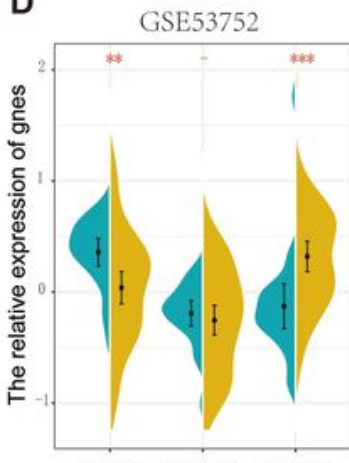

SUMO1 SUMO2 SUMO3
B

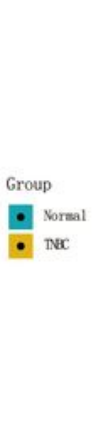

$\sqrt{2}$
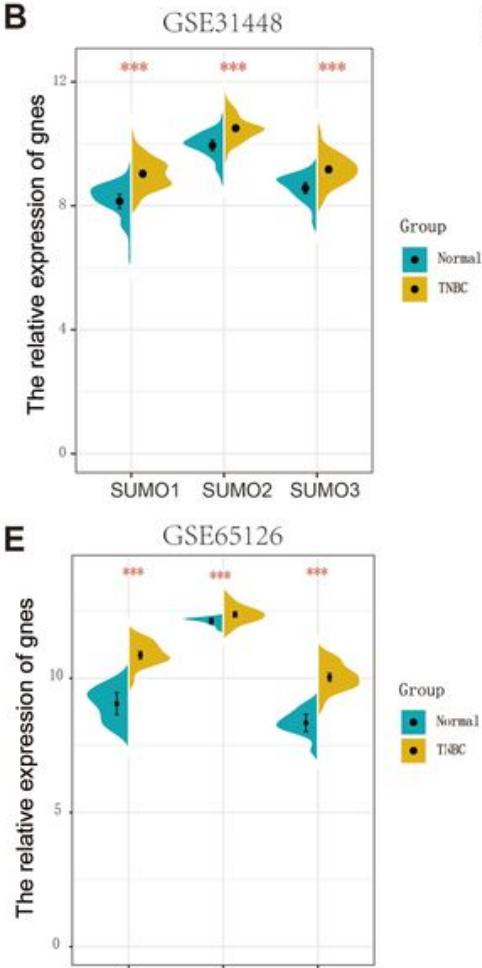

C

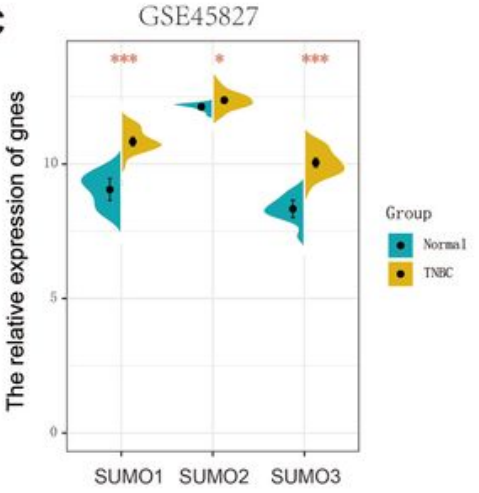

SUMO1 SUMO2 SUMO3

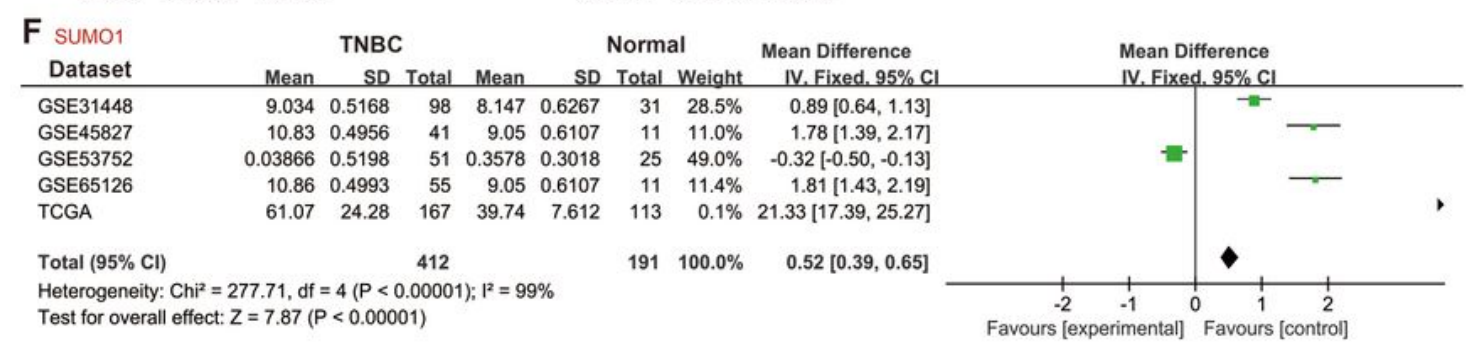

G sumo2

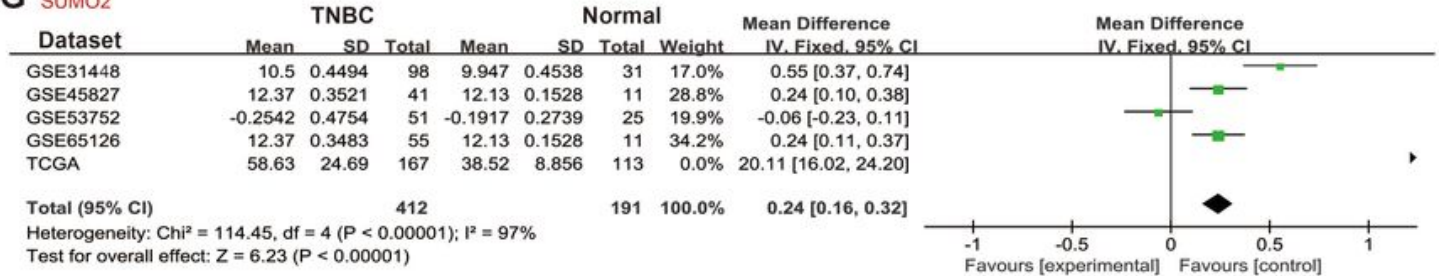
Test for overall effect: $Z=6.23(P<0.00001)$

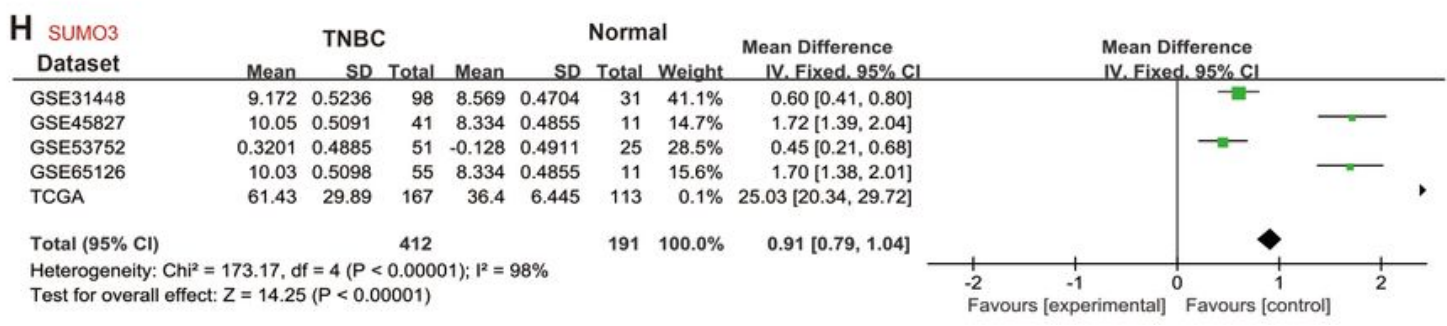

\section{Figure 2}

The mRNA expression of SUM01, SUMO2 and SUMO3 are up-regulated in TNBC. (A-E) The mRNA expression of SUM01, SUMO2 and SUMO3 in TCGA (A), GSE31448 (B), GSE45827 (C), GSE53752 (D) and GSE65126 (E). (F-H) Meta-analysis of SUM01 (F), SUMO2 (G) and SUMO3 (H) in TNBC patients based on TCGA, GSE31448, GSE45827, GSE53752 and GSE65216.-, $p>0.05 ; *, p<0.05 ; * \star, p<0.01 ; * \star \star$, $p<0.001$. 


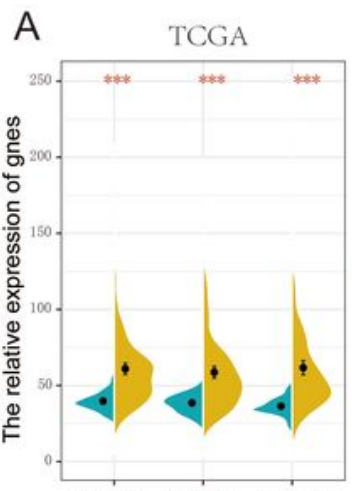

D

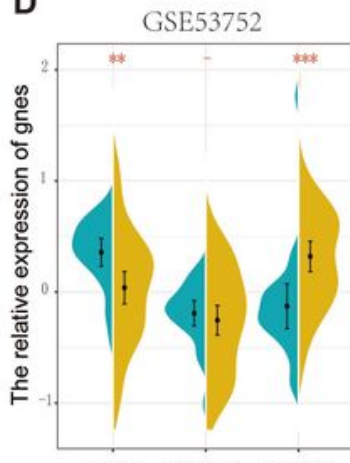

SUMO1 SUMO2 SUMO3
B

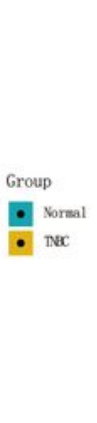

$\sqrt{2}$
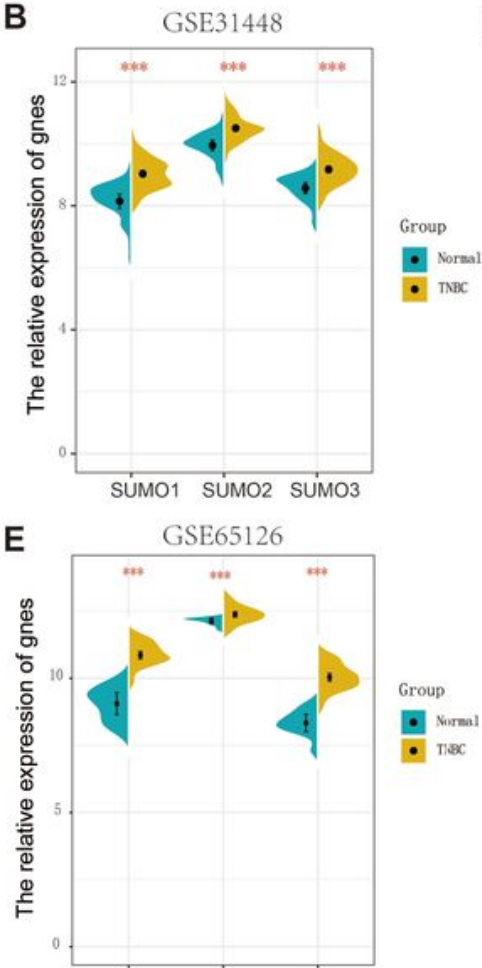

C

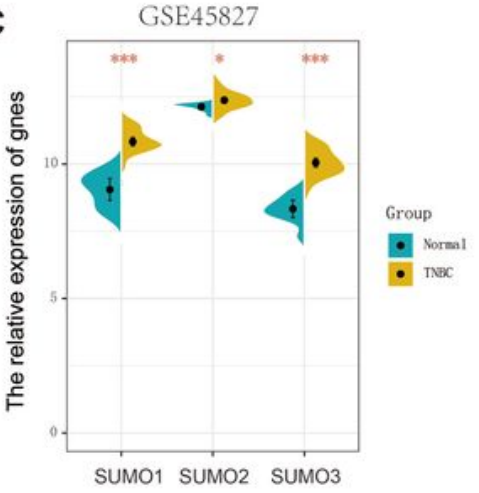

SUMO1 SUMO2 SUMO3

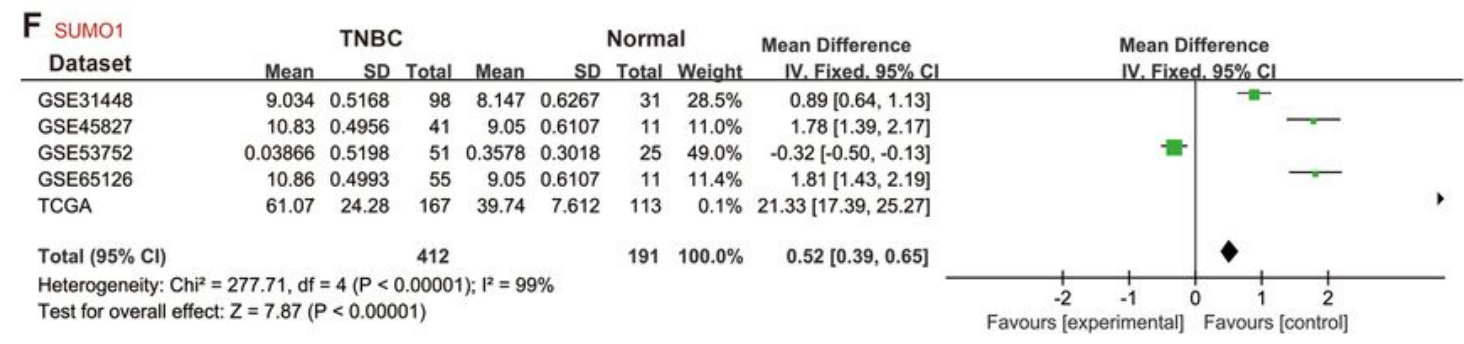

G sumo2

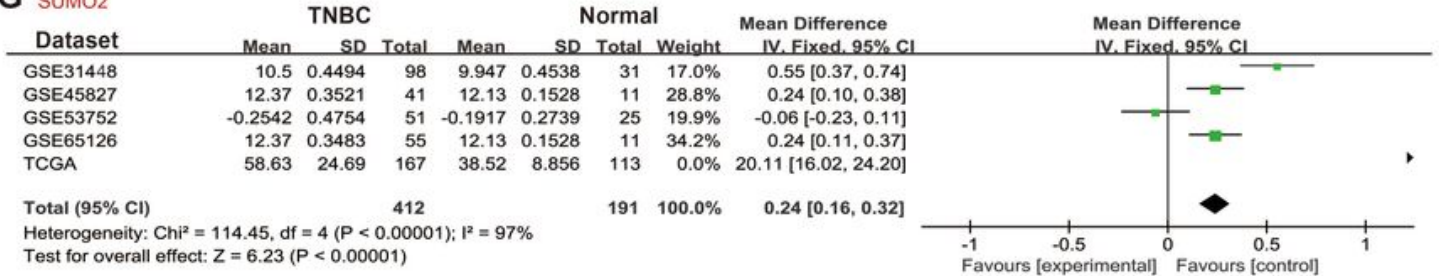
Test for overall effect: $Z=6.23(P<0.00001)$

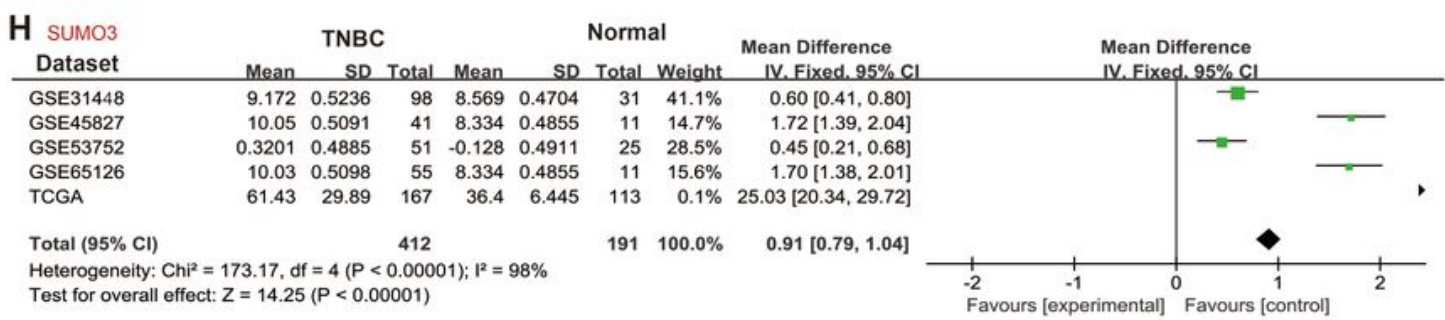

\section{Figure 2}

The mRNA expression of SUM01, SUMO2 and SUMO3 are up-regulated in TNBC. (A-E) The mRNA expression of SUM01, SUMO2 and SUMO3 in TCGA (A), GSE31448 (B), GSE45827 (C), GSE53752 (D) and GSE65126 (E). (F-H) Meta-analysis of SUM01 (F), SUMO2 (G) and SUMO3 (H) in TNBC patients based on TCGA, GSE31448, GSE45827, GSE53752 and GSE65216.-, $p>0.05 ; *, p<0.05 ; * \star, p<0.01 ; * \star \star$, $p<0.001$. 


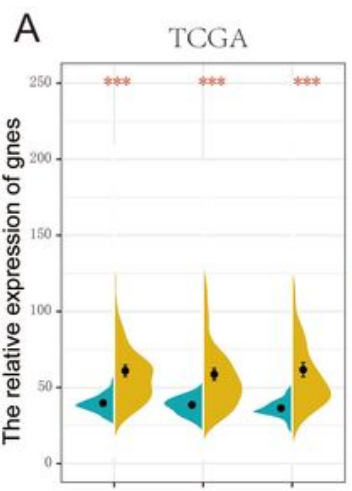

D

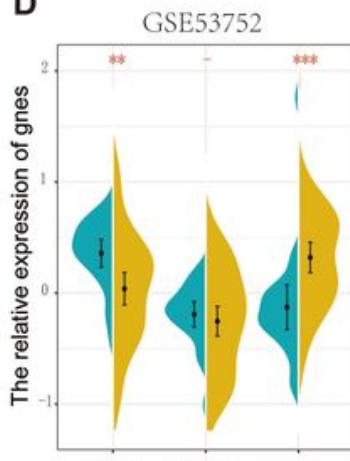

SUMO1 SUMO2 SUMO3
B

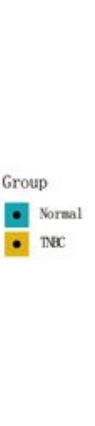

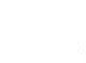
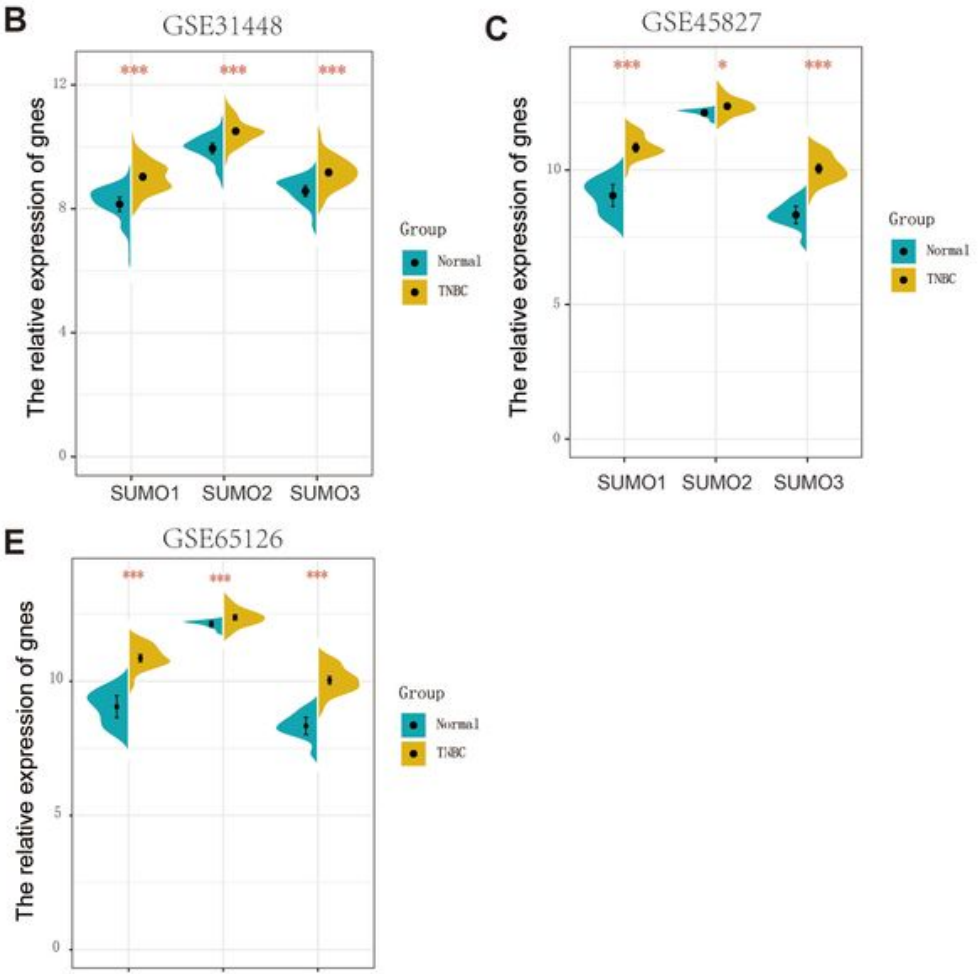

SUMO1 SUMO2 SUMO3

F sum01

TNBC

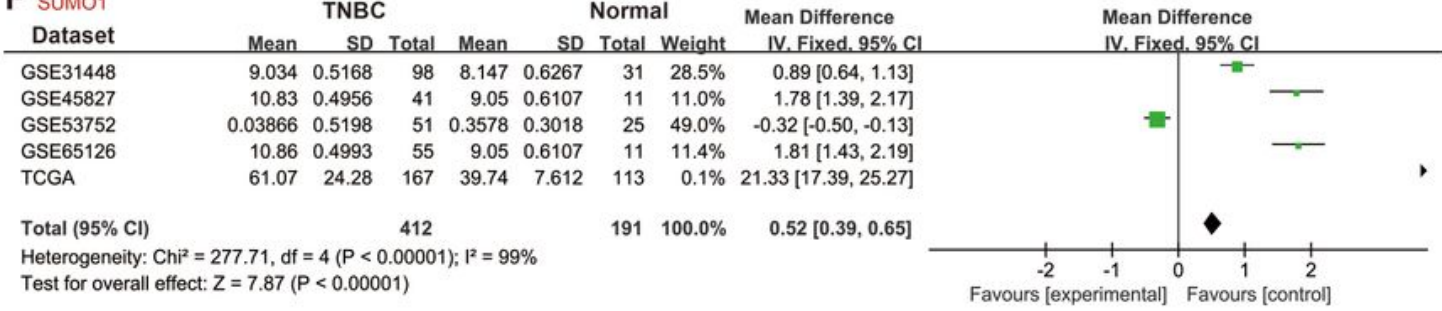

G sumo2

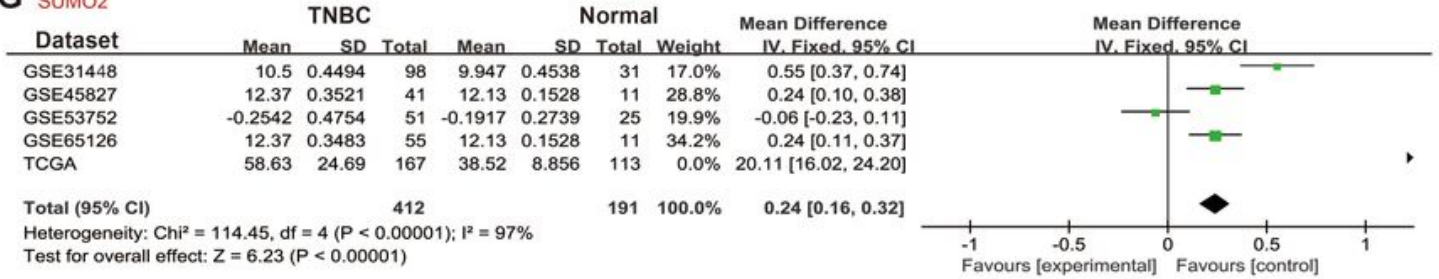

Test for overall effect: $Z=6.23(P<0.00001)$

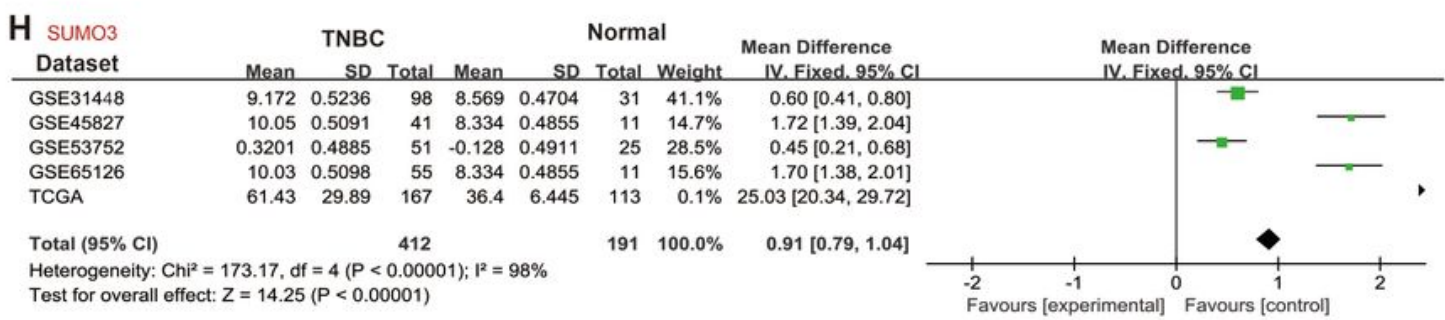

\section{Figure 2}

The mRNA expression of SUM01, SUMO2 and SUMO3 are up-regulated in TNBC. (A-E) The mRNA expression of SUM01, SUMO2 and SUMO3 in TCGA (A), GSE31448 (B), GSE45827 (C), GSE53752 (D) and GSE65126 (E). (F-H) Meta-analysis of SUM01 (F), SUMO2 (G) and SUMO3 (H) in TNBC patients based on TCGA, GSE31448, GSE45827, GSE53752 and GSE65216.-, $p>0.05 ; *, p<0.05 ; * \star, p<0.01 ; * \star \star$, $p<0.001$. 
A

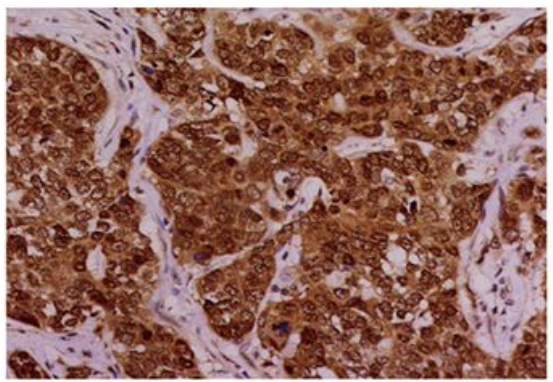

TNBC tissues

C

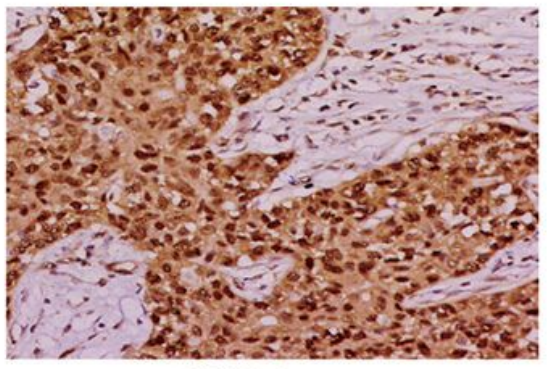

TNBC tissues

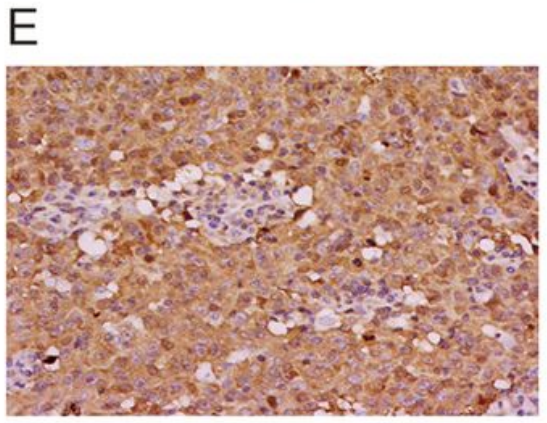

TNBC tissues

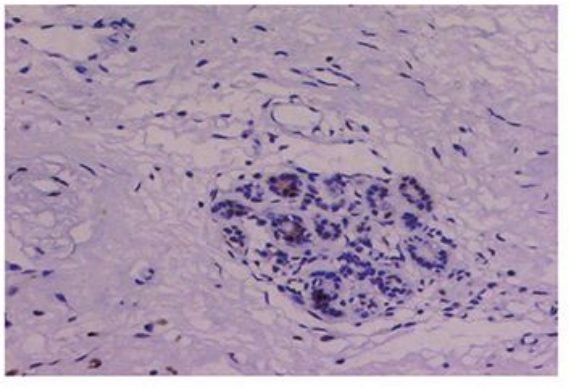

adjacent normal tissues

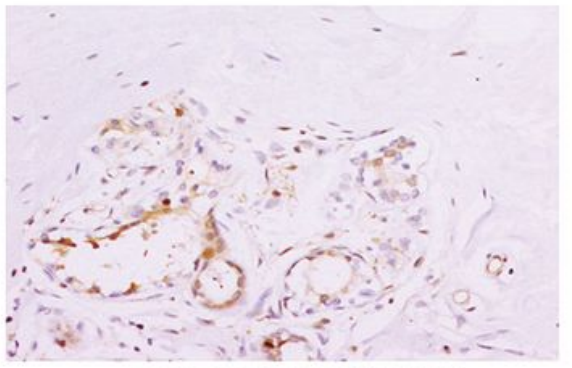

adjacent normal tissues

F

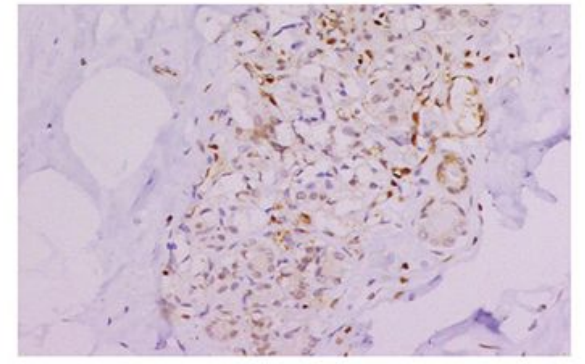

adjacent normal tissues

D
B
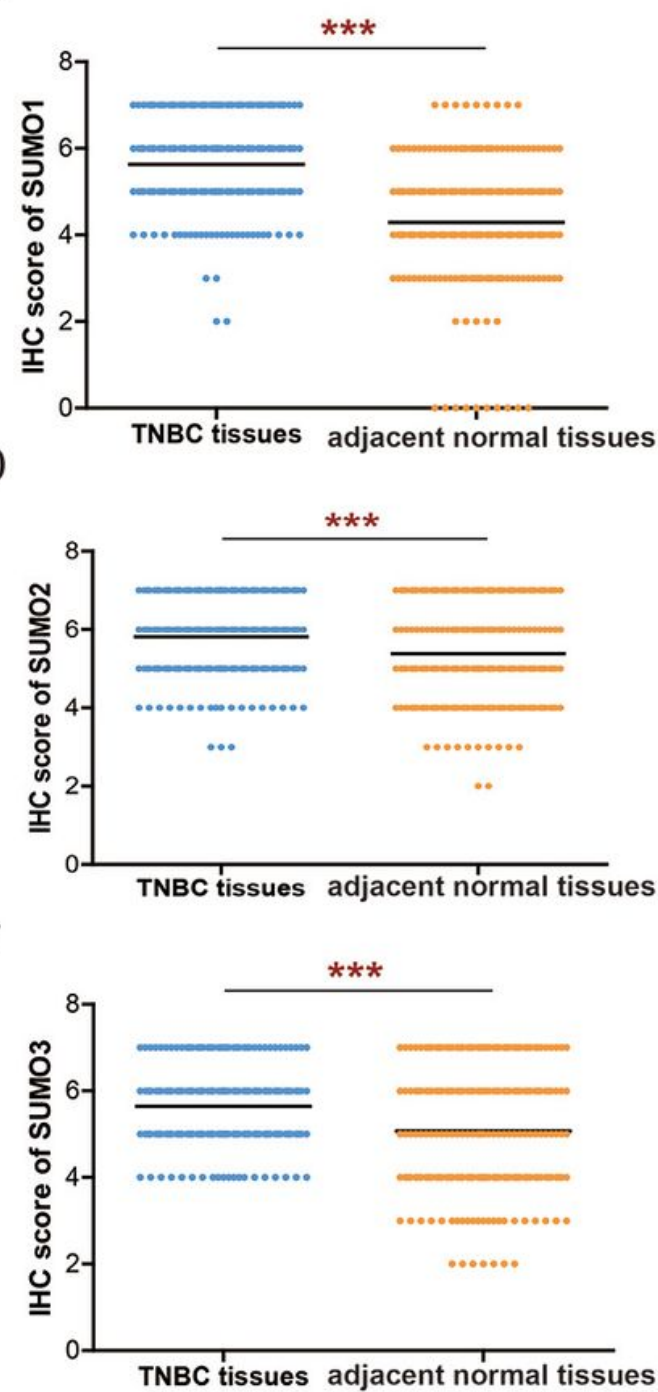

Figure 3

Immunohistochemical analyses of SUMO1, SUMO2 and SUMO3 proteins expression in TNBC (A) Representative immunohistochemical images of SUMO1 protein expression in TNBC tissues and adjacent normal tissues (magnification, $\times 200$ ). (B) Immunohistochemistry scores for SUM01 in TNBC patients. (C) Representative immunohistochemical images of SUMO2 protein expression in TNBC tissues and adjacent normal tissues (magnification, $\times 200$ ). (D) Immunohistochemistry scores for SUMO2 in TNBC patients. (E) Representative immunohistochemical images of SUMO3 protein expression in TNBC tissues and adjacent normal tissues (magnification, $\times 200$ ). (F) Immunohistochemistry scores for SUMO3 in TNBC patients. 
A

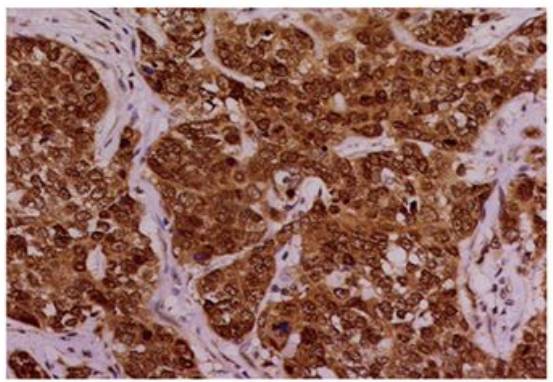

TNBC tissues

C

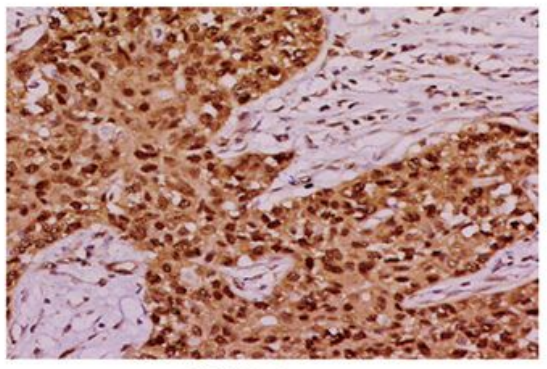

TNBC tissues

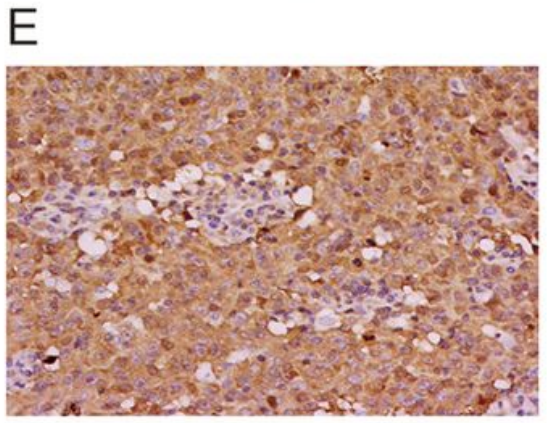

TNBC tissues

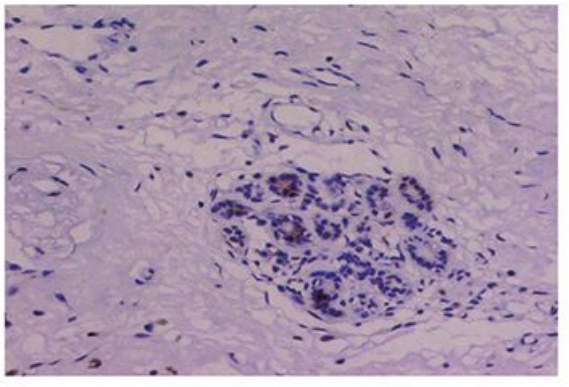

adjacent normal tissues

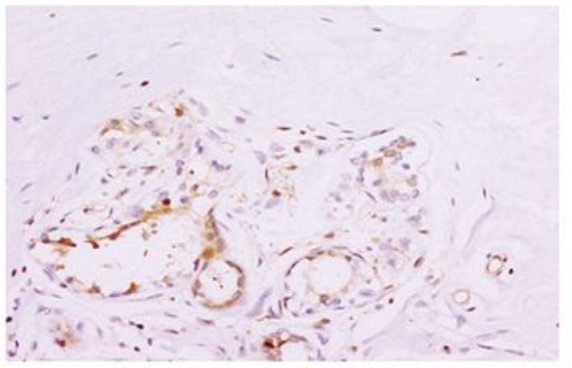

adjacent normal tissues

F

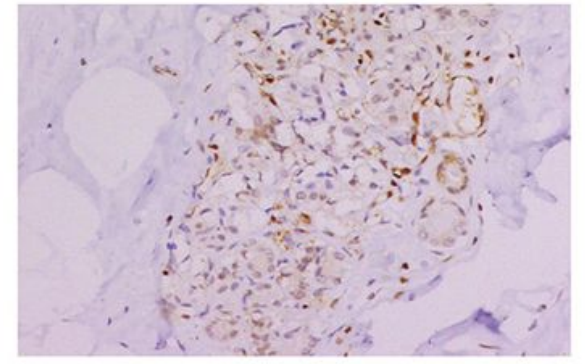

adjacent normal tissues

D
B
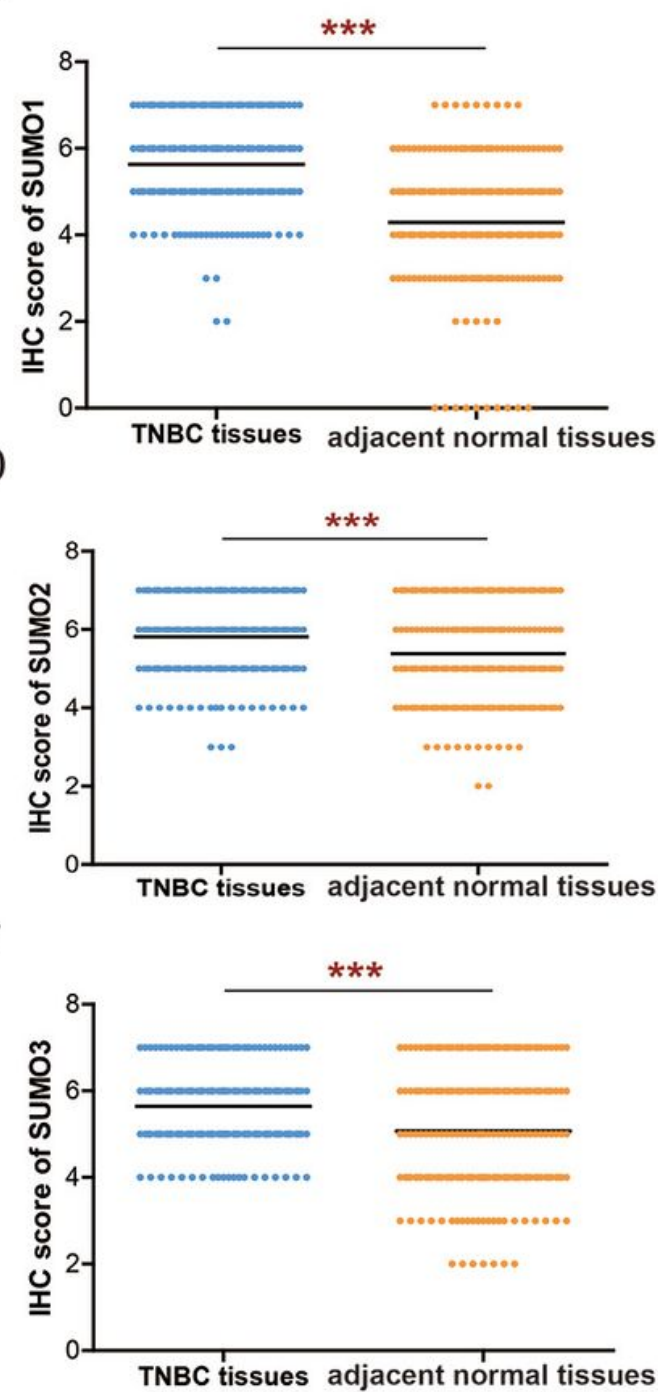

Figure 3

Immunohistochemical analyses of SUMO1, SUMO2 and SUMO3 proteins expression in TNBC (A) Representative immunohistochemical images of SUMO1 protein expression in TNBC tissues and adjacent normal tissues (magnification, $\times 200$ ). (B) Immunohistochemistry scores for SUM01 in TNBC patients. (C) Representative immunohistochemical images of SUMO2 protein expression in TNBC tissues and adjacent normal tissues (magnification, $\times 200$ ). (D) Immunohistochemistry scores for SUMO2 in TNBC patients. (E) Representative immunohistochemical images of SUMO3 protein expression in TNBC tissues and adjacent normal tissues (magnification, $\times 200$ ). (F) Immunohistochemistry scores for SUMO3 in TNBC patients. 
A

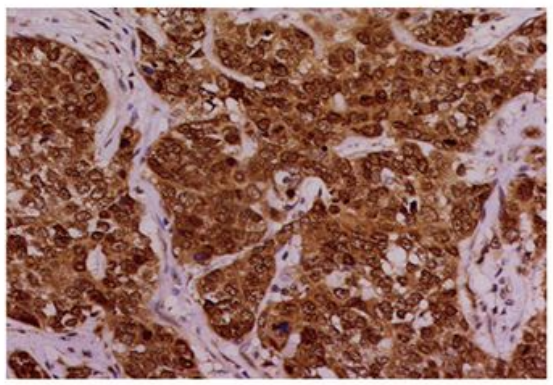

C

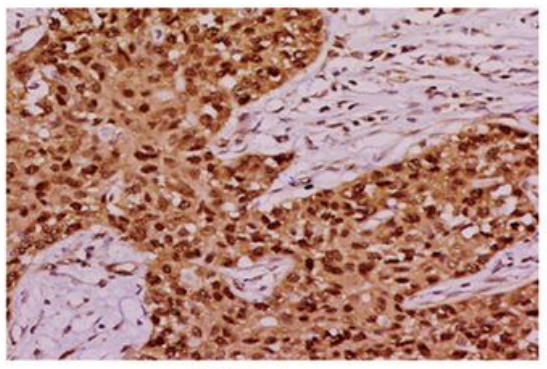

TNBC tissues

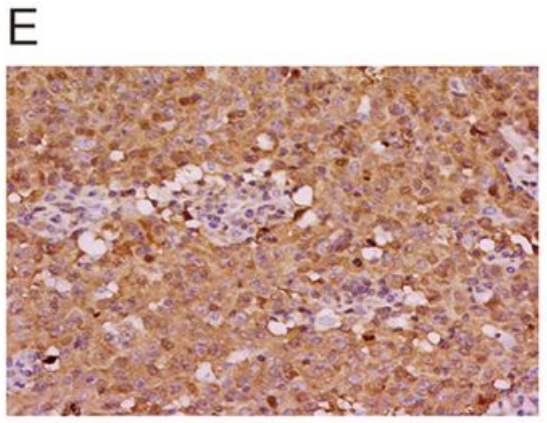

TNBC tissues

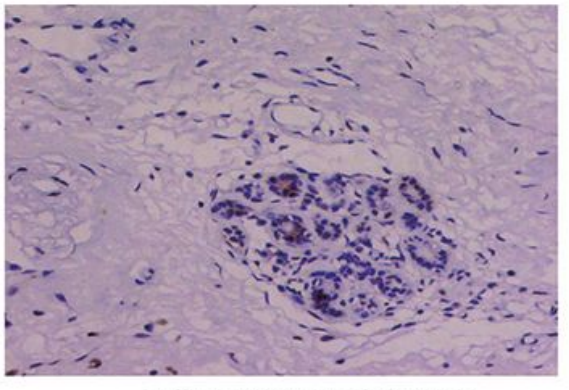

adjacent normal tissues

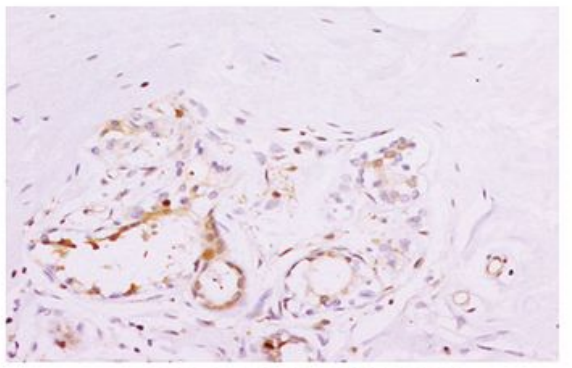

adjacent normal tissues

F

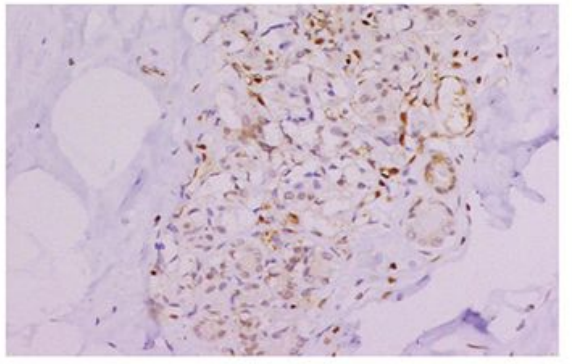

adjacent normal tissues

D
B
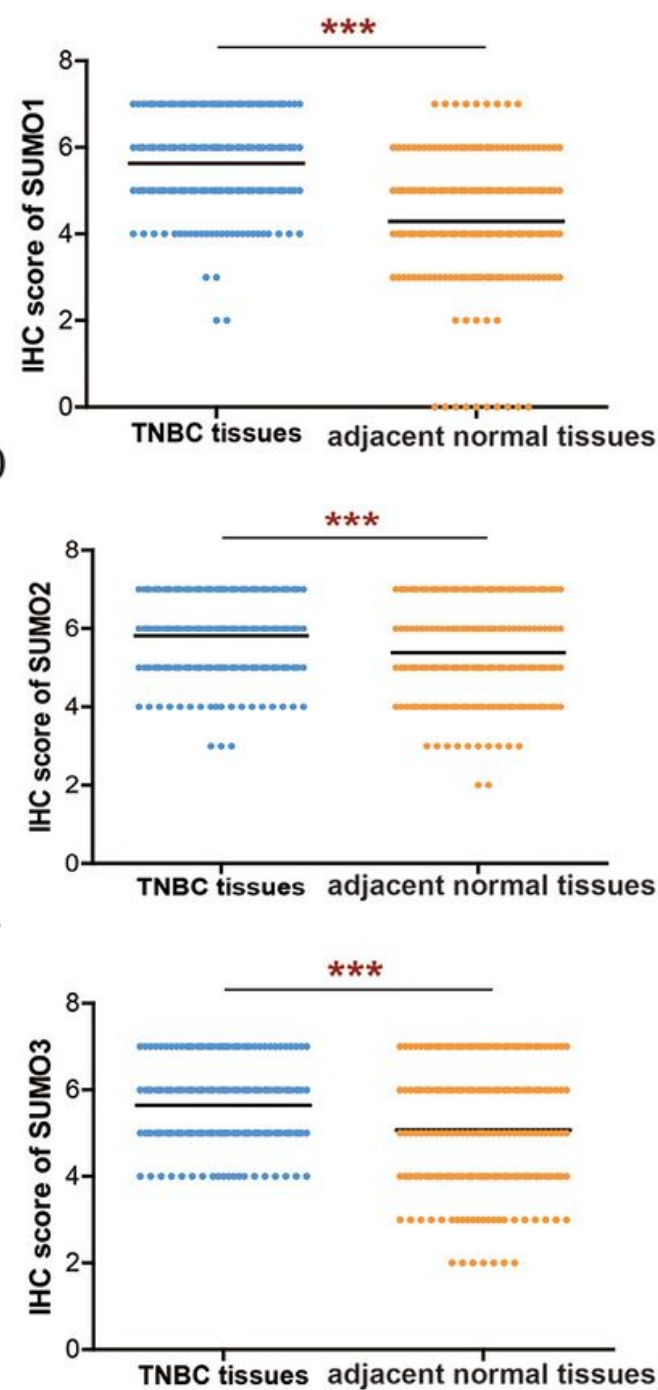

Figure 3

Immunohistochemical analyses of SUMO1, SUMO2 and SUMO3 proteins expression in TNBC (A) Representative immunohistochemical images of SUMO1 protein expression in TNBC tissues and adjacent normal tissues (magnification, $\times 200$ ). (B) Immunohistochemistry scores for SUM01 in TNBC patients. (C) Representative immunohistochemical images of SUMO2 protein expression in TNBC tissues and adjacent normal tissues (magnification, $\times 200$ ). (D) Immunohistochemistry scores for SUMO2 in TNBC patients. (E) Representative immunohistochemical images of SUMO3 protein expression in TNBC tissues and adjacent normal tissues (magnification, $\times 200$ ). (F) Immunohistochemistry scores for SUMO3 in TNBC patients. 

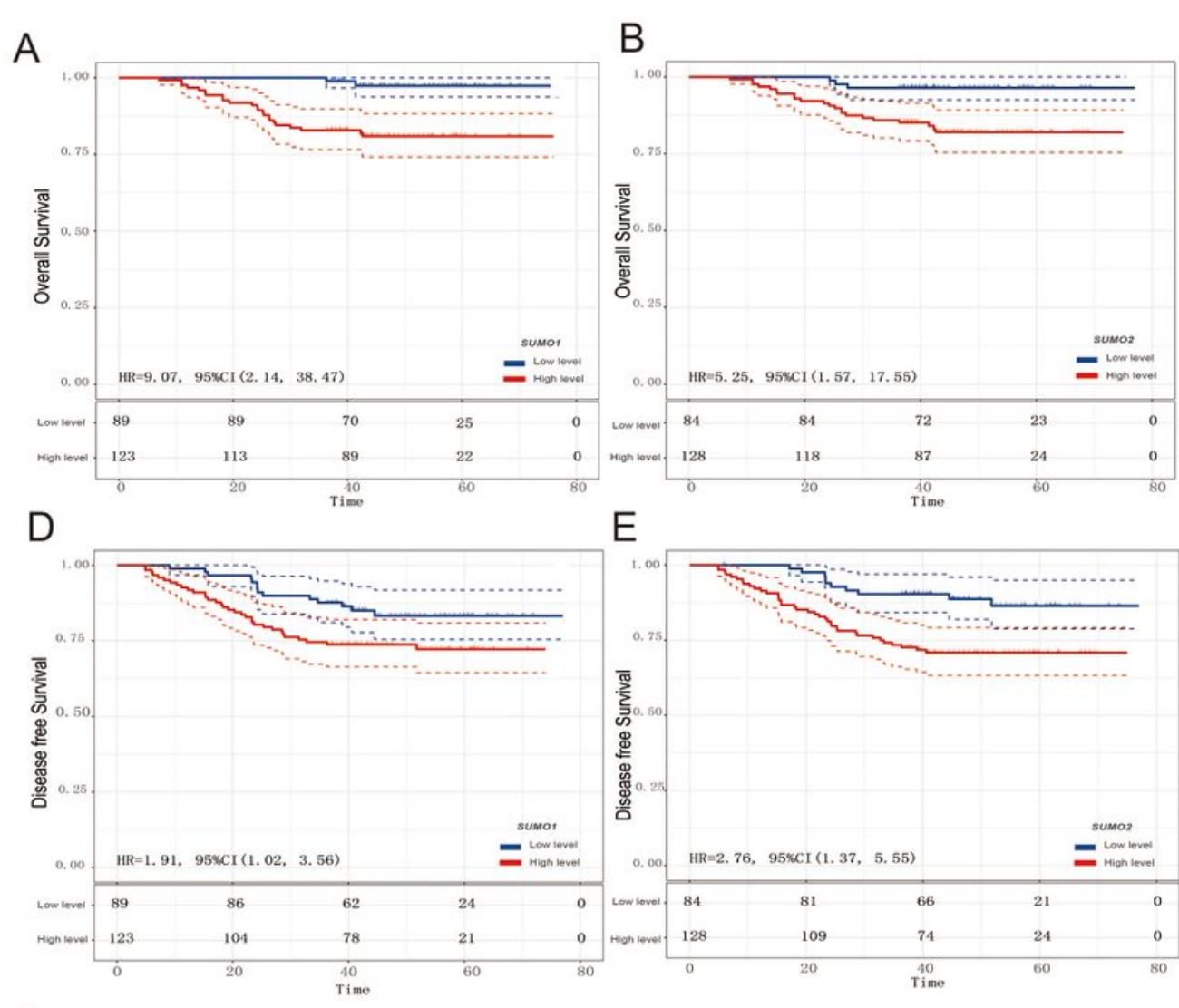

E
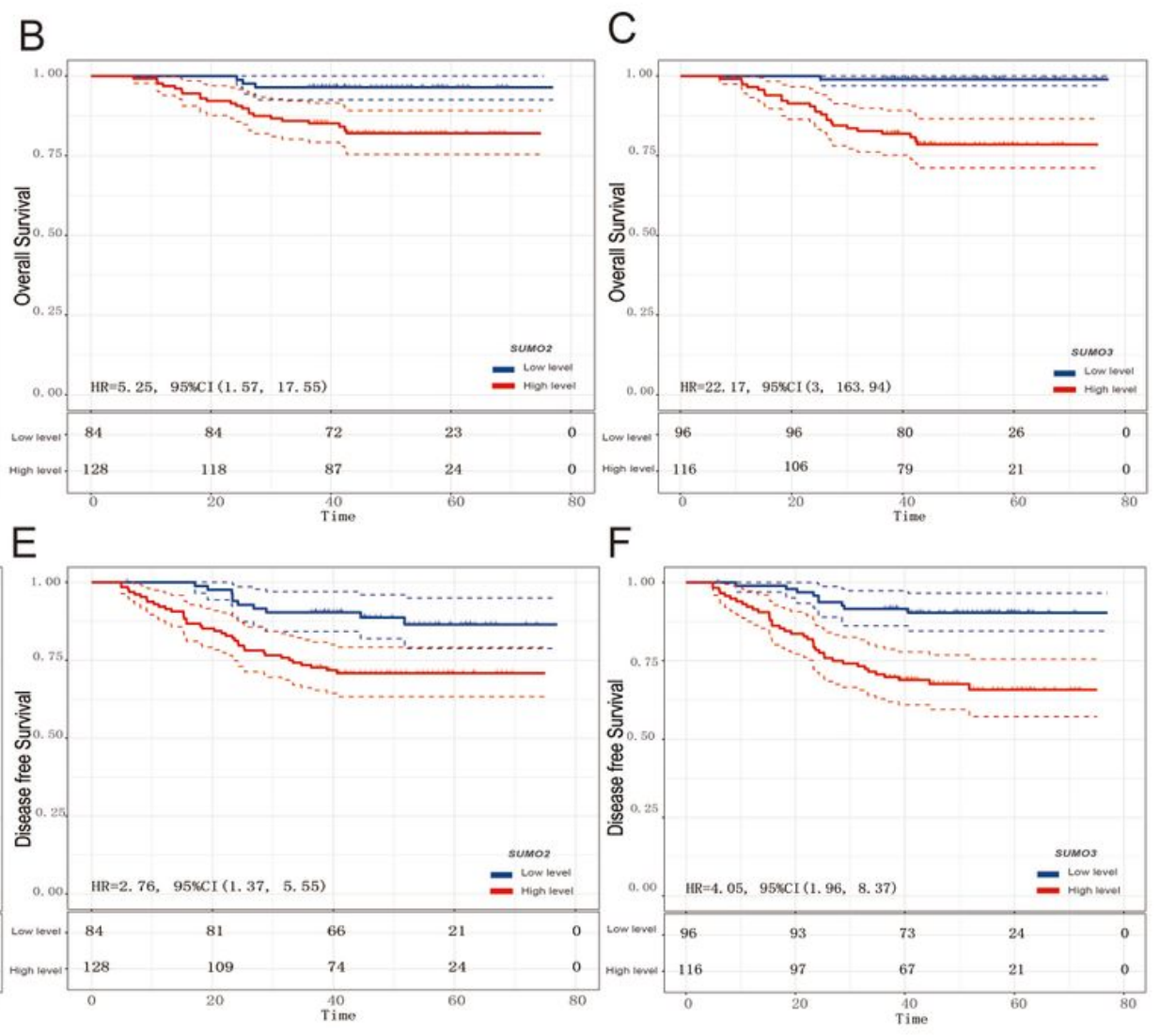

$\mathrm{F}$

\begin{tabular}{|c|c|c|c|c|c|c|c|c|c|c|c|c|c|}
\hline \multirow{3}{*}{ Variable } & & \multicolumn{6}{|c|}{ OS } & \multicolumn{6}{|c|}{ DFS } \\
\hline & & & \multicolumn{2}{|c|}{ Univariate analysis } & & \multirow{2}{*}{\multicolumn{2}{|c|}{$\begin{array}{l}\text { Multivariate analysis } \\
\text { pvalue Hazard Ratio(95\% Cl) }\end{array}$}} & & \multicolumn{2}{|c|}{ Univariate analysis } & & \multicolumn{2}{|c|}{ Multivariate analysis } \\
\hline & & & pvalue & Hazard Ratio(96\% Cl) & & & & & pvalue $\mathrm{r}$ & Hazard Ratio(96\% Cl) & & pvalue & Hazard Ratio(95\% Cl) \\
\hline Age & (>50 vs. 550 ) & - & 0.510 & $1.31(0.59 .291)$ & & & & 뭉 & 0.169 & $0.67(0.38,1.19)$ & & & \\
\hline Tumor size & (>2cm vs. $52 \mathrm{~cm})$ & 툴 1 & 0.124 & $2.06(0.82,5.15)$ & & & & 1. 를ㅅ..1 & 0.005 & $2.84(1.37,5.87)$ & $1 . \cdot \mathrm{E} \cdots \cdots, 1$ & 0.035 & $2.23(1.06,4.7)$ \\
\hline Lymph node metastasis & (yes vs. no) & $1 \cdot=\cdots \cdots \cdot 1$ & 0.000 & $5.73(2.15,15.26)$ & '…를 ……… & 0.001 & $9.57(2.68,34.18)$ & $1 \cdot \cdot \mathbf{n} \cdots \cdot \cdot 1$ & 0.000 & $3.53(1.89 .6 .6)$ & $1 \cdots=\cdots \cdots \cdot 1$ & 0.006 & $2.57(1.31,5.04)$ \\
\hline Tumor grade & (III vs. I+111) & = & 0.058 & $0.39(0.14,1.03)$ & & & & $1 \cdot=\cdots 1$ & 0.020 & $2.23(1.14,4.38)$ & $1 \cdot \ldots \cdots \cdots$ & 0.020 & $2.25(1.14,4.45)$ \\
\hline Lymphovascular invasion & (yes vs. no) & 口. & 0.005 & $0.32(0.14,0.71)$ & 由 & 0.640 & $1.22(0.53 .228)$ & 1. 불…1 & 0.000 & $3.37(1.88 .6 .05)$ & $1 \cdot=‥ 6$ & 0.023 & $2.08(1.11,3.89)$ \\
\hline Breast conserving surgery & (Des vs.no) & $1 \cdot .1$ & 0.797 & $0.77(0.1,5.68)$ & & & & 1ㅂ.ㄹ…1 & 0.540 & $1.44(0.45,4.64)$ & & & \\
\hline Radiotherapy & (no vs. yes) & {$[\cdots$} & 0.044 & $3(1,03,8,76)$ & 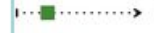 & 0.004 & $7.71(1.94,30.62)$ & $1 \cdot$ 둘…1 & 0.053 & $2.33(0.99 .5 .49)$ & & & \\
\hline sUMO1 & (high vs, low) & 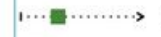 & 0.003 & $9.06(2.14,38.46)$ & 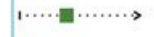 & 0.002 & $11.25(2.52 .50 .14)$ & $1=\sqrt{n} \cdot-4$ & 0.043 & $1.91(1.02,3.56)$ & $1 \cdot=\cdots \cdot$ & 0.056 & $1.88(0.98,3.49)$ \\
\hline SUMO2 & (Migh vs. low) & 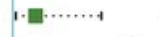 & 0.007 & $5.25(1.57,17.54)$ & $\mid=… 1$ & 0.036 & $3.68(1.09,12.4)$ & $1 \cdot=\sqrt{n} \cdots \cdot 1$ & 0.004 & $2.7 \Theta(1.37,5.55)$ & $1 \cdot=\cdots \cdot 1$ & 0.057 & $2.02(0.98,4.16)$ \\
\hline sUMO3 & (high vs. low) & 1............. & 0.002 & $22.17(3,163.88)$ & 1.............. $=$ & 0.003 & $22.36(2.78,179.87)$ & 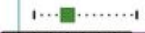 & 0.000 & $4.05(1.96,8.37)$ & 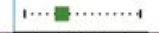 & 0.002 & $3.17(1.5,6.7)$ \\
\hline
\end{tabular}

\section{Figure 4}

High expression levels of SUMO1, SUMO2 and SUMO3 proteins are associated with the poor prognosis of TNBC patients with. (A-C) Kaplan-Meier survival analysis showing the impact of SUMO1 (A), SUMO2 (B) and SUMO3 (C) protein expression on OS. (D-F) Kaplan-Meier survival analysis showing the impact of SUMO1 (D), SUMO2 (E) and SUMO3 (F) protein expression on disease free survival. 『G囚Univariate and multivariate Cox regression analyses. 

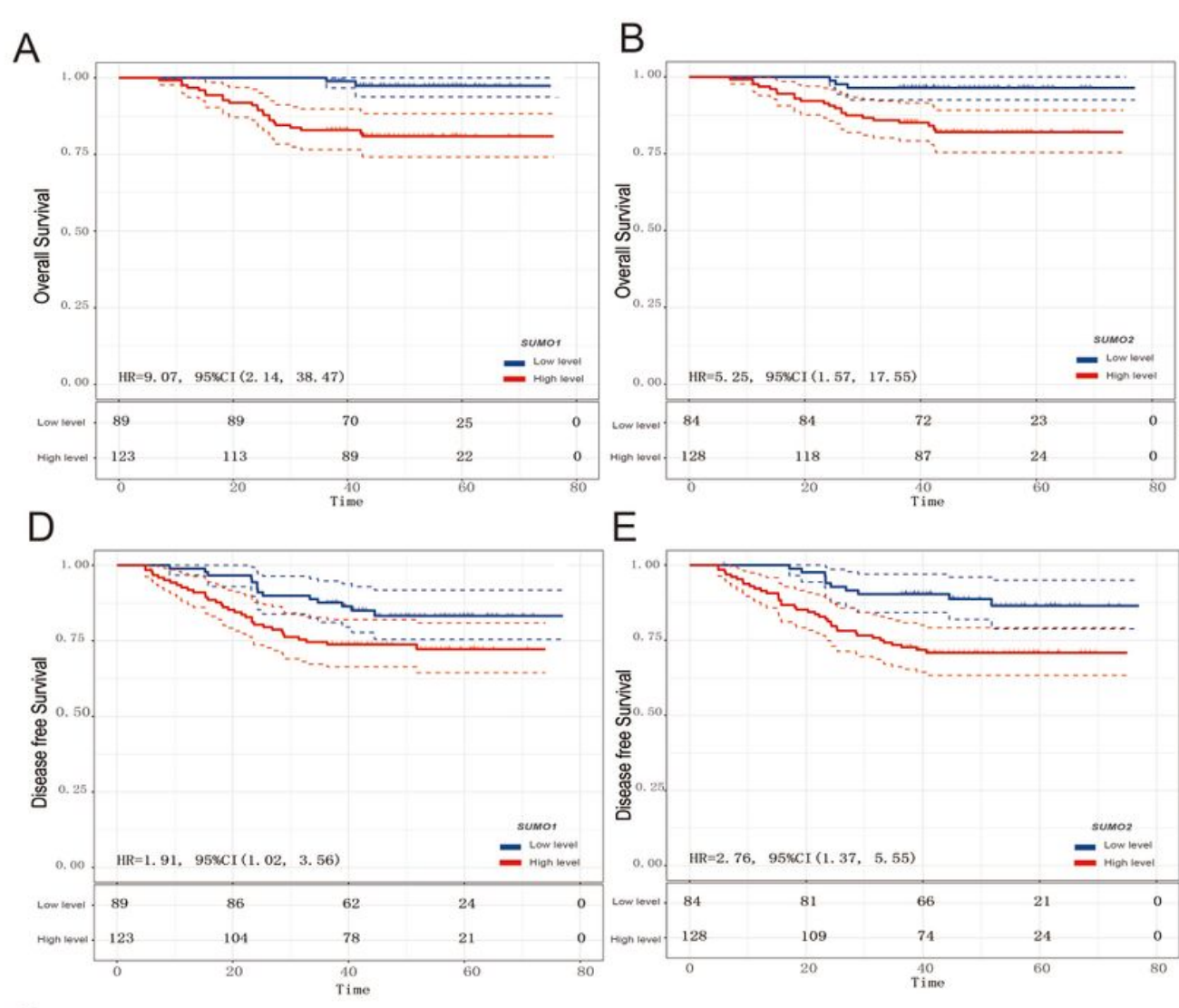

E
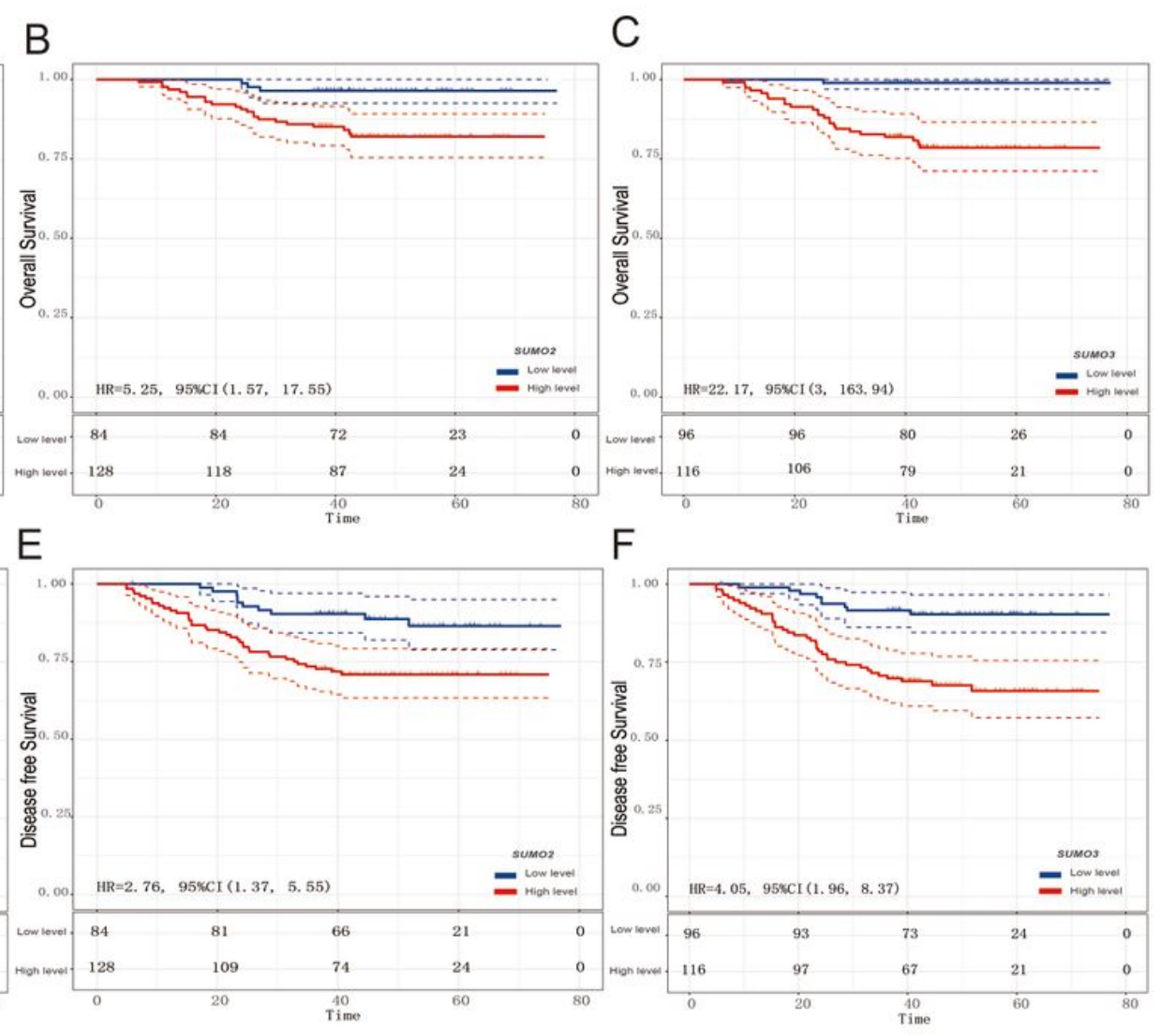

$\mathrm{F}$

\begin{tabular}{|c|c|c|c|c|c|c|c|c|c|c|c|c|c|}
\hline \multirow{3}{*}{ Variable } & & \multicolumn{6}{|c|}{ OS } & \multicolumn{6}{|c|}{ DFS } \\
\hline & & & \multicolumn{2}{|c|}{ Univariate analysis } & & \multirow{2}{*}{\multicolumn{2}{|c|}{$\begin{array}{l}\text { Multivariate analysis } \\
\text { pvalue Hazard Ratio(95\% Cl) }\end{array}$}} & & \multicolumn{2}{|c|}{ Univariate analysis } & & \multicolumn{2}{|c|}{ Multivariate analysis } \\
\hline & & & pvalue & Hazard Ratio(96\% Cl) & & & & & pvalue & Hazard Ratio(96\% Cl) & & pvalue & Hazard Ratio(95\% Cl) \\
\hline Age & (>50 vs. 550 ) & - & 0.510 & $1.31(0.59 .2 .91)$ & & & & 둥 & 0.169 & $0.67(0.38,1.19)$ & & & \\
\hline Tumor size & ( $>2 \mathrm{~cm}$ vs. $52 \mathrm{~cm})$ & 툴 & 0.124 & $2.06(0.82 .5 .15)$ & & & & 1. 플…1 & 0.005 & $2.84(1.37,5.87)$ & 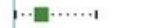 & 0.035 & $223(1.06,4.7)$ \\
\hline Lymph node metastasis & (yes vs. no) & 1. & 0.000 & $5.73(2.15,15.26)$ & 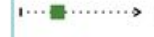 & 0.001 & $9.57(2.68,34.18)$ & 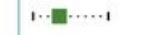 & 0.000 & $3.53(1.89 .6 .6)$ & 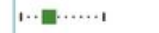 & 0.006 & $2.57(1.31,5.04)$ \\
\hline Tumor grade & (III vs. I+11) & ㅁ & 0.058 & $0.39(0.14,1.03)$ & & & & $1 \cdot=\cdots 1$ & 0.020 & $2.23(1.14,4.38)$ & $1, \cdot=\cdots \cdot 1$ & 0.020 & $2.25(1.14,4.45)$ \\
\hline Lymphovascular invasion & (yes vs. no) & - & 0.005 & $0.32(0.14,0.71)$ & 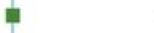 & 0.640 & $1.22(0.53,2.28)$ & $1 \cdot$ 불…1 & 0.000 & $3.37(1.88 .6 .05)$ & $1 \cdot=\cdots \cdot 1$ & 0.023 & $2.08(1.11,3.89)$ \\
\hline Breast consenving surgery & (yes vs. no) & $\nabla \cdot \cdots 1$ & 0.797 & $0.77(0.1,5.68)$ & & & & 1 1 ……1 & 0.540 & $1.44(0.45,4.64)$ & & & \\
\hline Radiotherapy & (novs. yes) & 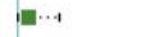 & 0.044 & $3(1.03,8.76)$ & 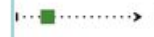 & 0.004 & $7.71(1.94,30.62)$ & $1 \cdot$ 를…1 & 0.053 & $2.33(0.99 .5 .49)$ & & & \\
\hline sumo1 & (high vs, low) & 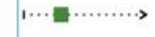 & 0.003 & $9.06(2.14,38.46)$ & 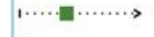 & 0.002 & $11.25(2.52 .50 .14)$ & $1=\sqrt{n} \cdot 4$ & 0.043 & $1.91(1.02,3.56)$ & $1 \cdot$ anct & 0.056 & $1.86(0.98,3.49)$ \\
\hline SUMO2 & (Migh vs. llow) & 1. & 0.007 & $5.25(1.57,17.54)$ & 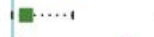 & 0.036 & $3.68(1.09,12.4)$ & $1 \cdot=\sqrt{n} \cdots \cdot 1$ & 0.004 & $2.76(1.37,5.55)$ & $1 \cdot=\sqrt{n} \cdots \cdot 4$ & 0.057 & $2.02(0.98,4.16)$ \\
\hline SUMO3 & (high vs. low) & 1.............. & 0.002 & $22.17(3,163.88)$ & 1.............. $=$ & 0.003 & $22.36(2.78,179.87)$ & 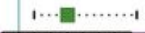 & 0.000 & $4.05(1.96,8.37)$ & 1…를……… & 0.002 & $3.17(1.5,6.7)$ \\
\hline
\end{tabular}

\section{Figure 4}

High expression levels of SUMO1, SUMO2 and SUMO3 proteins are associated with the poor prognosis of TNBC patients with. (A-C) Kaplan-Meier survival analysis showing the impact of SUMO1 (A), SUMO2 (B) and SUMO3 (C) protein expression on OS. (D-F) Kaplan-Meier survival analysis showing the impact of SUMO1 (D), SUMO2 (E) and SUMO3 (F) protein expression on disease free survival. 『G囚Univariate and multivariate Cox regression analyses. 

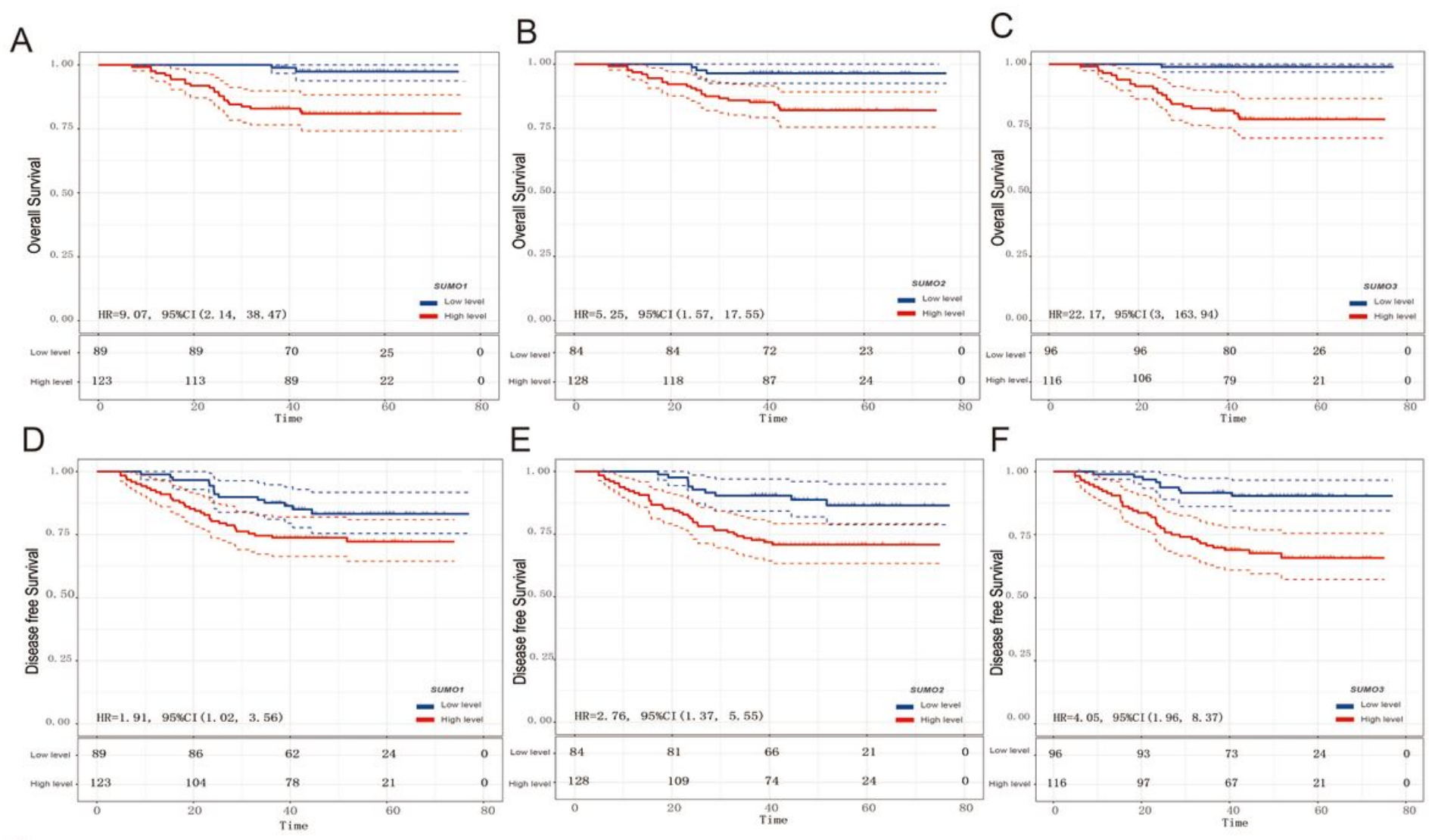

G

\begin{tabular}{|c|c|c|c|c|c|c|c|}
\hline \multirow{3}{*}{ Variable } & & \multicolumn{6}{|c|}{ OS } \\
\hline & & & \multicolumn{2}{|c|}{ Univariate analysis } & & \multirow{2}{*}{\multicolumn{2}{|c|}{$\begin{array}{l}\text { Multivariate analysis } \\
\text { pvalue Hazard Ratio(95\% cl) }\end{array}$}} \\
\hline & & & pvalue & Hazard Ratio(95\% Cl) & & & \\
\hline Age & (>50 vs. 550 ) & - & 0.510 & $1.31(0.59 .291)$ & & & \\
\hline Tumor size & ( $22 \mathrm{~cm}$ vs. $52 \mathrm{~cm})$ & 툴 & 0.124 & $2.06(0.82 .5 .15)$ & & & \\
\hline Lymph node metastasis & (yes vs. no) & 1. & 0.000 & $5.73(2.15,15.26)$ & '…를 ……… & 0.001 & $9.57(2.68 .34 .18)$ \\
\hline Tumor grade & (III vs. I+111) & a & 0.058 & $0.39(0.14,1.03)$ & & & \\
\hline Lymphovascular invasion & (yes vs. no) & - & 0.005 & $0.32(0.14,0.71)$ & 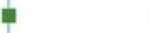 & 0.640 & $1.22(0.53,2.28)$ \\
\hline Breast conserving surgery & (yes vs. no) & $\nabla \cdot \cdot 1$ & 0.797 & $0.77(0.1 .5 .68)$ & & & \\
\hline Radiotherapy & (novs. yes) & $=\cdots-1$ & 0.044 & $3(1.03,8.76)$ & 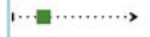 & 0.004 & $7.71(1.94,30.62)$ \\
\hline sUMO1 & (high vs, low) & 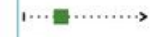 & 0.003 & $9.06(2.14,38.46)$ & $\mid \cdots \cdots=1 \cdots \cdots$, & 0.002 & $11.25(2.52 .50 .14)$ \\
\hline sUMO2 & (Migh vs. low) & 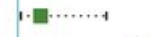 & 0.007 & $5.25(1.57,17.54)$ & $1=$ & 0.036 & $3.68(1.09,12.4)$ \\
\hline SUMO3 & (high vs. low) & 1……....... & 0.002 & $22.17(3,163.88)$ & 1.............. & 0.003 & $22.36(2.78,179.87)$ \\
\hline
\end{tabular}

\begin{tabular}{|c|c|c|c|c|c|}
\hline \multicolumn{6}{|c|}{ DFS } \\
\hline & \multicolumn{2}{|c|}{ Univariate analysis } & & \multicolumn{2}{|c|}{ Multivariate analysis } \\
\hline & pvalue & Hazard Ratio(96\% Cl) & & pvalue & Hazard Ratio(95\% Cl) \\
\hline 름 & 0.169 & $0.67(0.38,1.19)$ & & & \\
\hline 1. 블…1 & 0.005 & $2.84(1.37,5.87)$ & 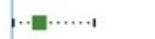 & 0.035 & $223(1.06,4.7)$ \\
\hline 1... & 0.000 & $3.53(1.89 .6 .6)$ & 1...……1 & 0.006 & $2.57(1.31,5.54)$ \\
\hline $1=\cdots 1$ & 0.020 & $2.23(1.14,4.38)$ & 1... $=\cdots \cdot 1$ & 0.020 & $2.25(1.14 .4 .45)$ \\
\hline $1 \cdot=\ldots \cdot 1$ & 0.000 & $3.37(1.88 .6 .05)$ & $1 \cdot=‥ 64$ & 0.023 & $2.08(1.11,3.89)$ \\
\hline 1ㄹ. …1 & 0.540 & $1.44(0.45,4.64)$ & & & \\
\hline 1. 둘…1 & 0.053 & $2.33(0.99 .5 .49)$ & & & \\
\hline $1=4$ & 0.043 & $1.91(1.02,3.56)$ & 1. E....1 & 0.056 & $1.86(0.98,3.49)$ \\
\hline $1 \cdot$ 들 …1 & 0.004 & $2.76(1.37,5.55)$ & $1 \cdot-\frac{1}{2} \cdots \cdot 4$ & 0.057 & $2.02(0.98,4.16)$ \\
\hline 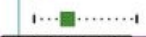 & 0.000 & $4.05(1.06,8.37)$ & $1 \cdots=\sqrt{n} \cdot \cdots \cdots \cdots \cdot 1$ & 0.002 & $3.17(1.5 .6 .7)$ \\
\hline
\end{tabular}

\section{Figure 4}

High expression levels of SUMO1, SUMO2 and SUMO3 proteins are associated with the poor prognosis of TNBC patients with. (A-C) Kaplan-Meier survival analysis showing the impact of SUMO1 (A), SUMO2 (B) and SUMO3 (C) protein expression on OS. (D-F) Kaplan-Meier survival analysis showing the impact of SUMO1 (D), SUMO2 (E) and SUMO3 (F) protein expression on disease free survival. 『G囚Univariate and multivariate Cox regression analyses. 

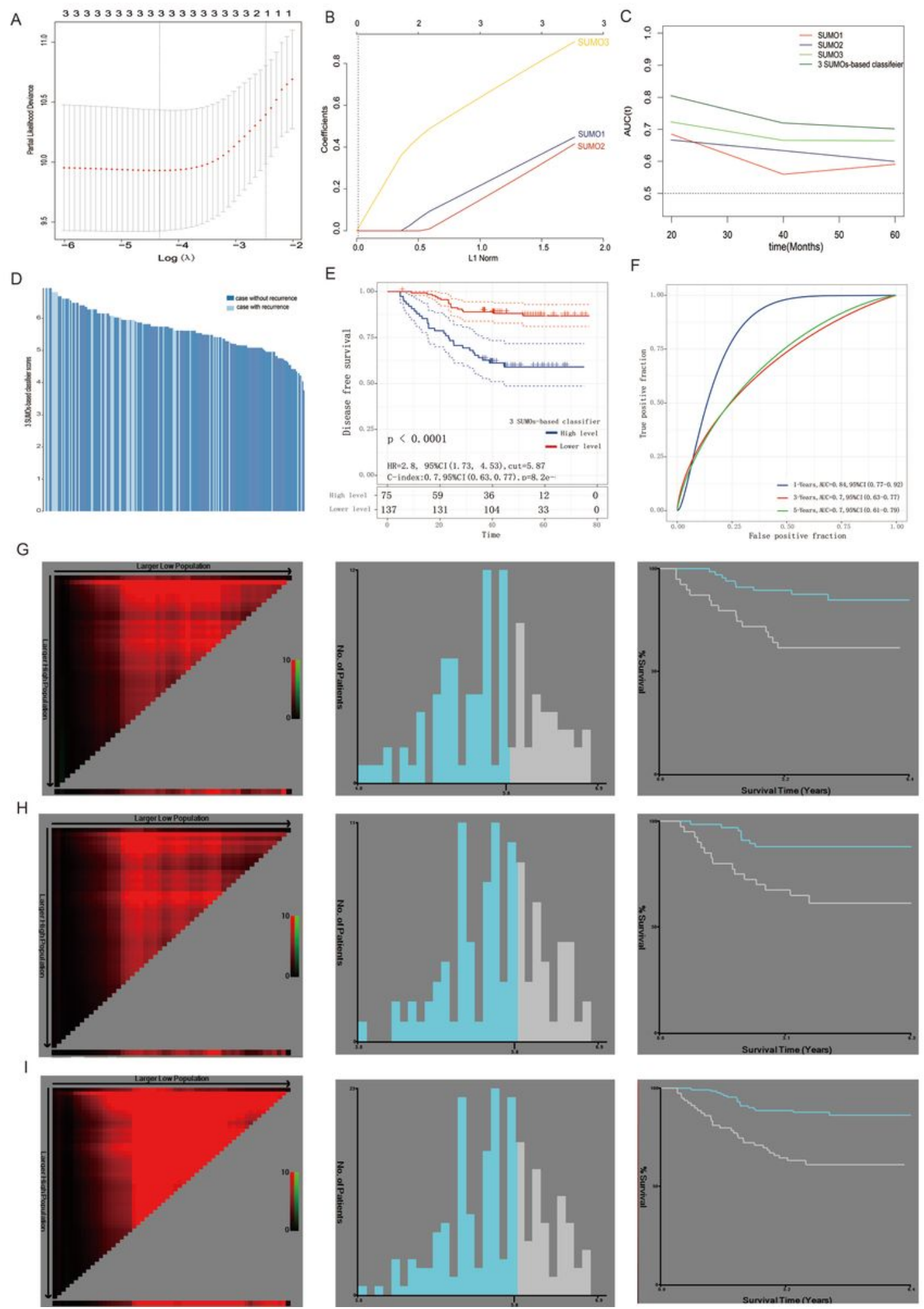

\section{Figure 5}

Construction of 3 SUMOs-based classifier to predict disease free survival in TNBC patients (A) Partial likelihood deviance of DFS for the LASSO coefficient profiles. (B) LASSO coefficient profiles of the SUM01/2/3 proteins for DFS. (C) Time-dependent ROC (receiver operator characteristic) curves compare the prognostic accuracy of the 3 SUMOs-based classifier with clinicopathological risk factors and single SUMOs in all 212 TNBC patients. (D) Risk score by the 3 SUMOs-based classifier in recurrence and 
without recurrence patients. (E) Kaplan-Meier survival analysis showing the impact of 3 SUMOs-based classifier on OS. (F) Time-dependent ROC curves compare the prognostic accuracy of the 3 SUMOs-based classifier with clinicopathological risk factors in all 212 TNBC patients used AUCs at 1, 3, and 5 years to assess prognostic accuracy. (G)Training cohort. (H) Validation cohort. (I) Total cohort.
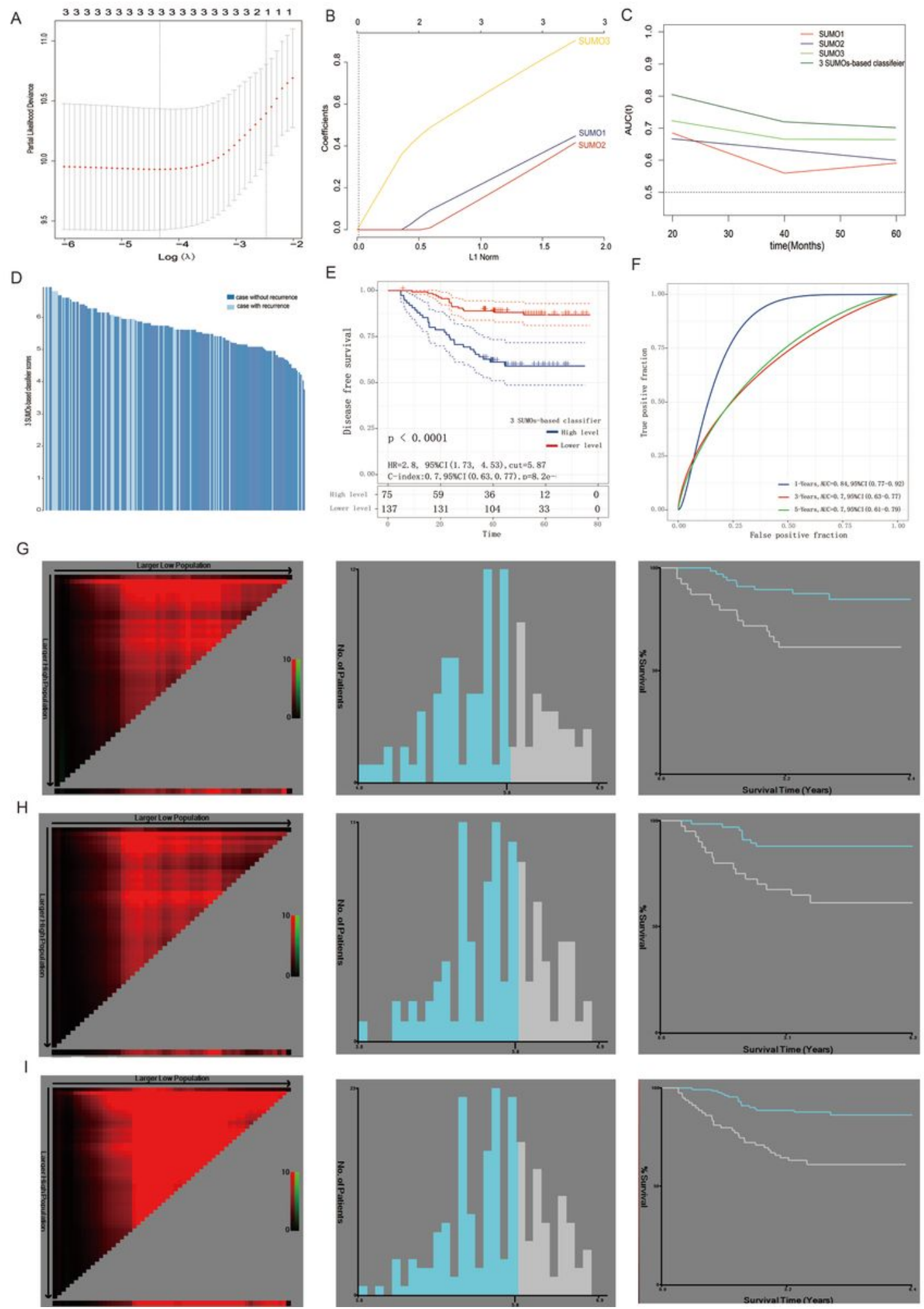

Figure 5 
Construction of 3 SUMOs-based classifier to predict disease free survival in TNBC patients (A) Partial likelihood deviance of DFS for the LASSO coefficient profiles. (B) LASSO coefficient profiles of the SUM01/2/3 proteins for DFS. (C) Time-dependent ROC (receiver operator characteristic) curves compare the prognostic accuracy of the 3 SUMOs-based classifier with clinicopathological risk factors and single SUMOs in all 212 TNBC patients. (D) Risk score by the 3 SUMOs-based classifier in recurrence and without recurrence patients. (E) Kaplan-Meier survival analysis showing the impact of 3 SUMOs-based classifier on OS. (F) Time-dependent ROC curves compare the prognostic accuracy of the 3 SUMOs-based classifier with clinicopathological risk factors in all 212 TNBC patients used AUCs at 1, 3, and 5 years to assess prognostic accuracy. (G)Training cohort. (H) Validation cohort. (I) Total cohort. 

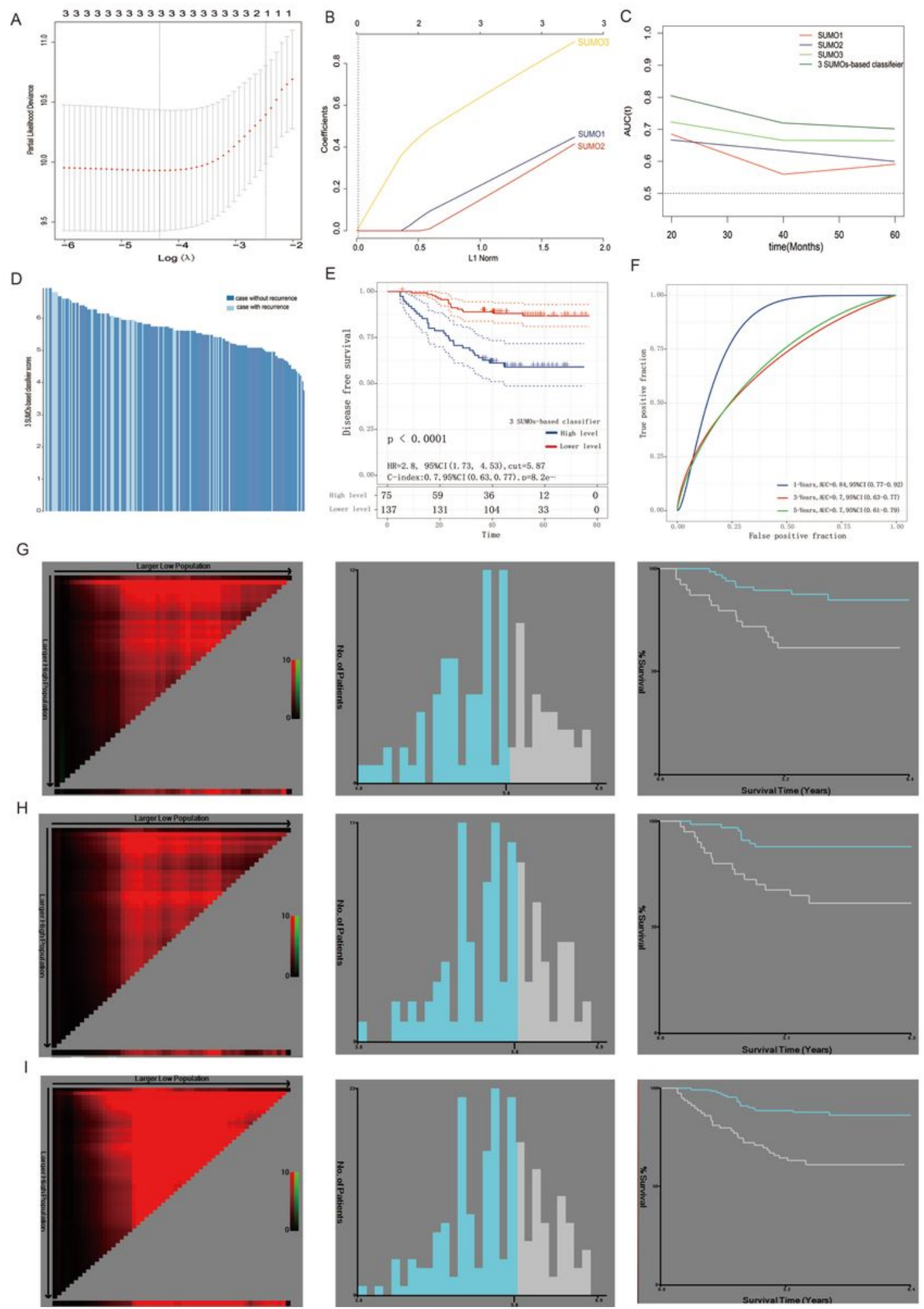

\section{Figure 5}

Construction of 3 SUMOs-based classifier to predict disease free survival in TNBC patients (A) Partial likelihood deviance of DFS for the LASSO coefficient profiles. (B) LASSO coefficient profiles of the SUM01/2/3 proteins for DFS. (C) Time-dependent ROC (receiver operator characteristic) curves compare the prognostic accuracy of the 3 SUMOs-based classifier with clinicopathological risk factors and single SUMOs in all 212 TNBC patients. (D) Risk score by the 3 SUMOs-based classifier in recurrence and 
without recurrence patients. (E) Kaplan-Meier survival analysis showing the impact of 3 SUMOs-based classifier on OS. (F) Time-dependent ROC curves compare the prognostic accuracy of the 3 SUMOs-based classifier with clinicopathological risk factors in all 212 TNBC patients used AUCs at 1, 3, and 5 years to assess prognostic accuracy. (G)Training cohort. (H) Validation cohort. (I) Total cohort.

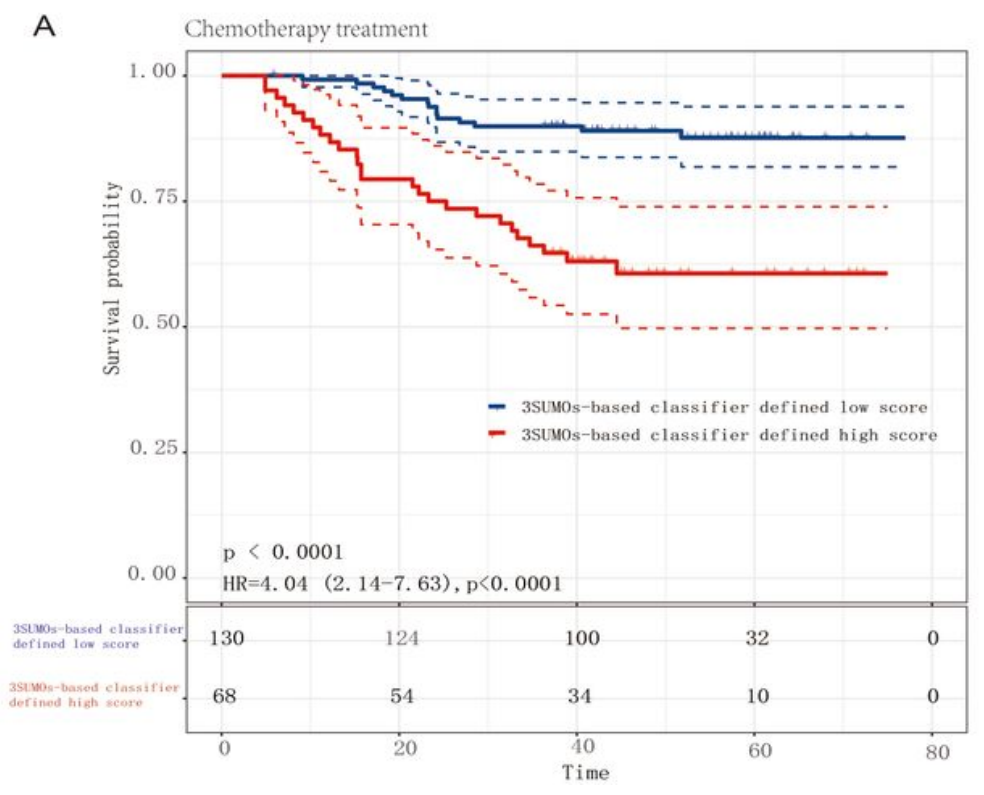

B

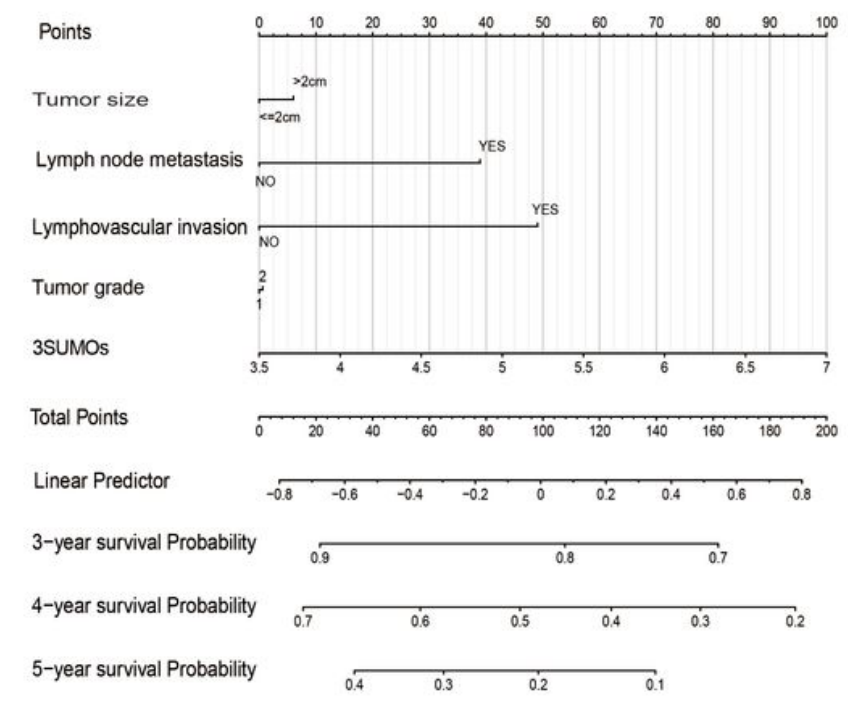

Figure 6

Nomograms to predict risk of tumor recurrence for TNBC patient with chemotherapy (A) Kaplan-Meier survival curves for patients in TNBC with chemotherapy. (B) Nomograms for predicting proportion of patients with DFS in TNBC patients with chemotherapy.
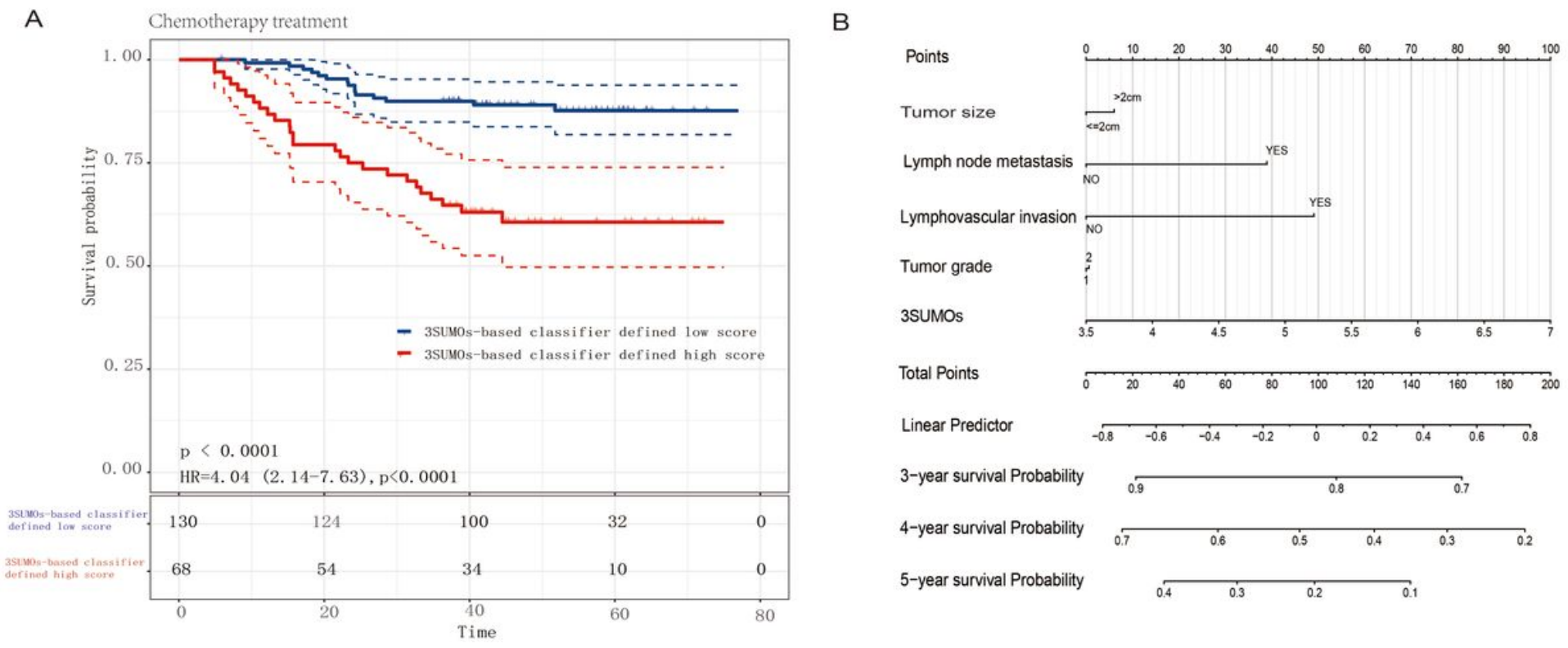

Figure 6

Nomograms to predict risk of tumor recurrence for TNBC patient with chemotherapy (A) Kaplan-Meier survival curves for patients in TNBC with chemotherapy. (B) Nomograms for predicting proportion of 
patients with DFS in TNBC patients with chemotherapy.
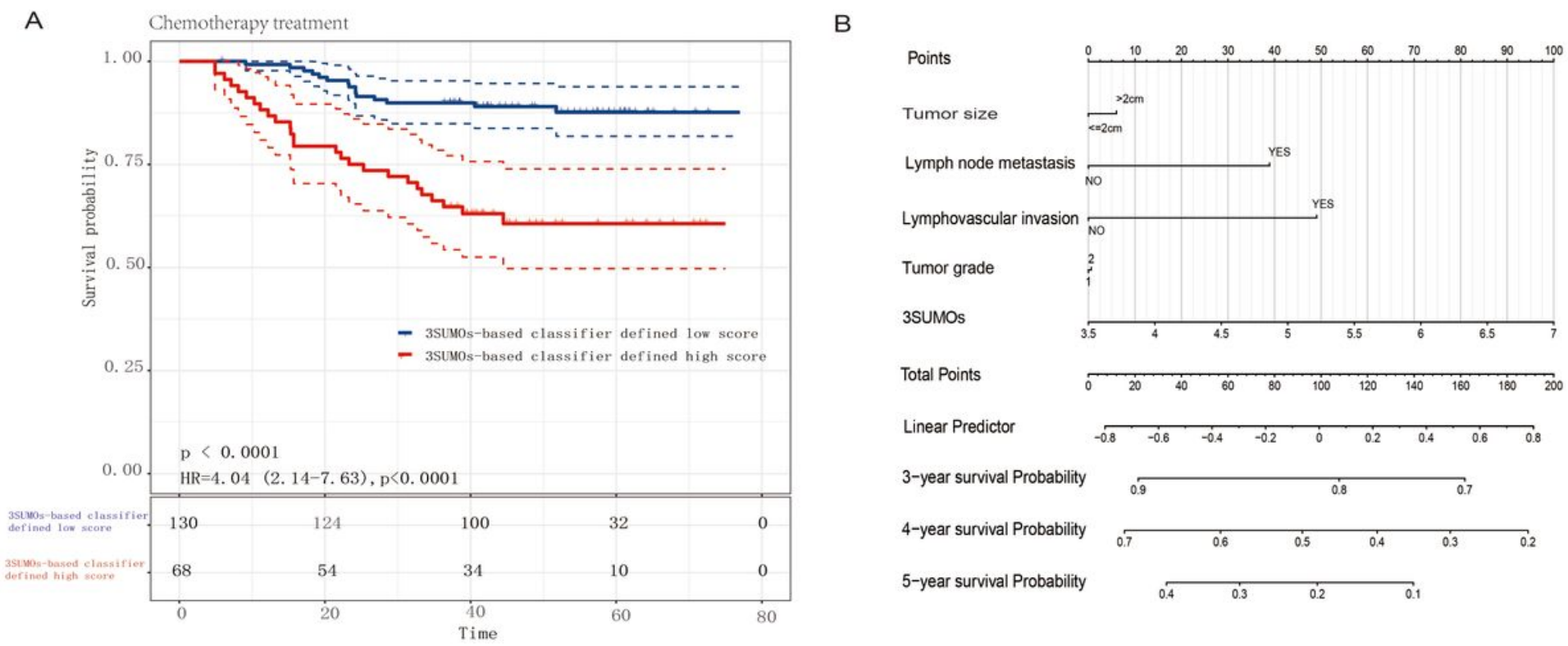

\section{Figure 6}

Nomograms to predict risk of tumor recurrence for TNBC patient with chemotherapy (A) Kaplan-Meier survival curves for patients in TNBC with chemotherapy. (B) Nomograms for predicting proportion of patients with DFS in TNBC patients with chemotherapy. 

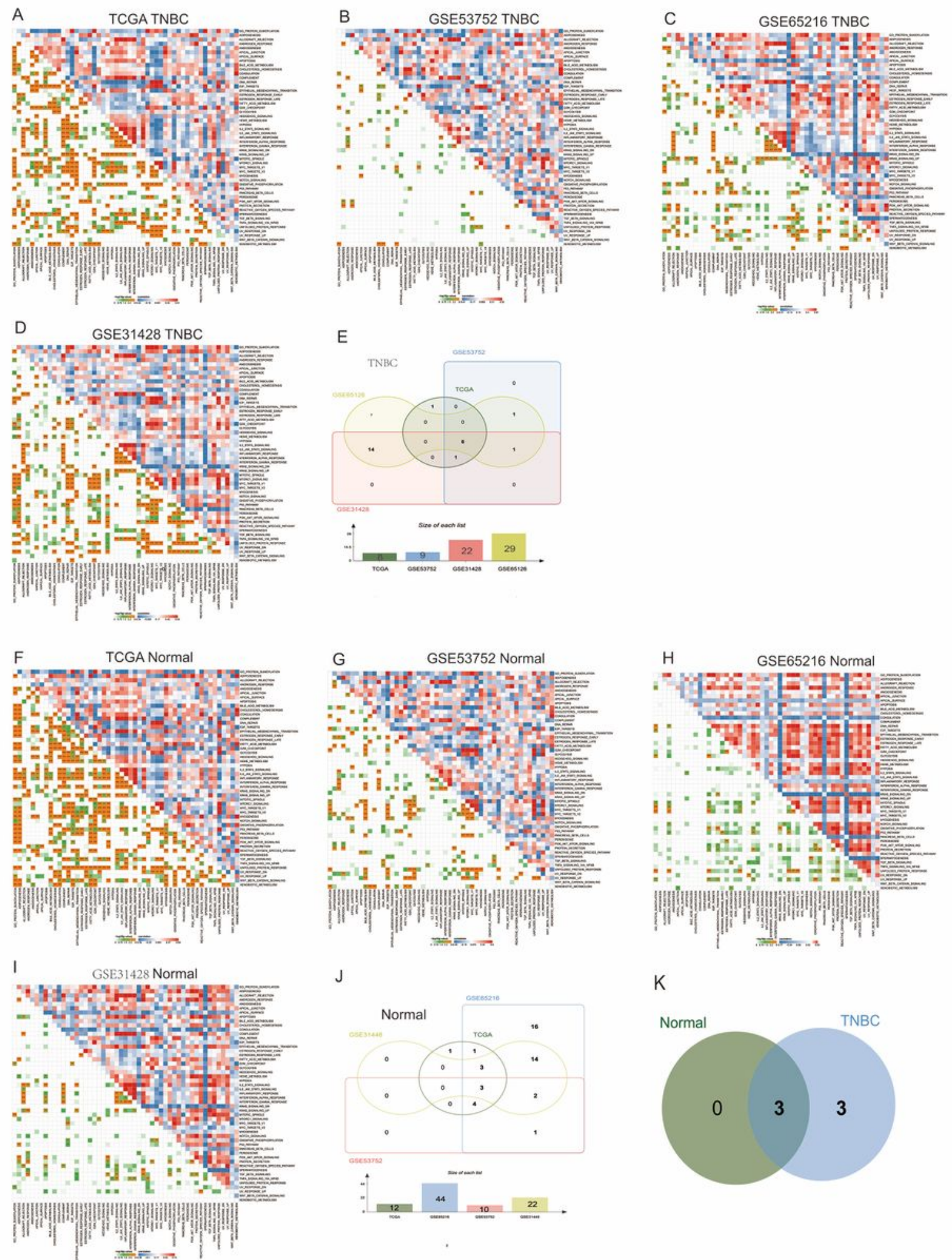

$J$

K
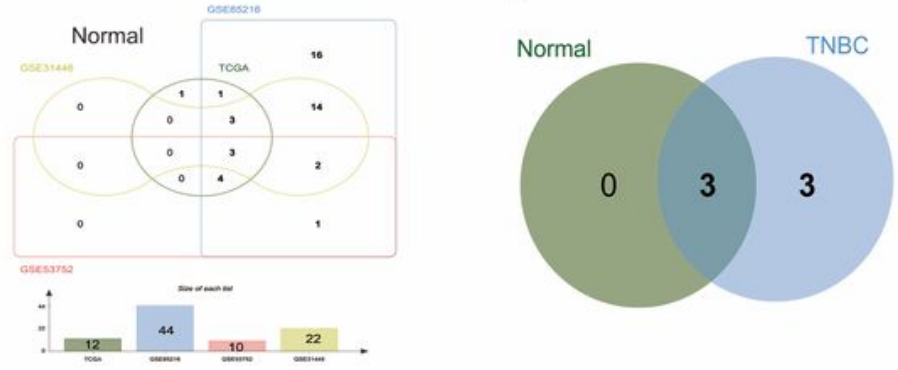

\section{Figure 7}

The positive correlation between SUMOylation and E2F-targets, MYC-targets-V1, Mtorc1-signaling, Mitotic-spindle, G2M-checkpoint and Unfolded protein response in TNBC tumor tissues (A-D) Correlation between the expression of SUMOylation and 50 oncogenic pathways in TNBC tumor tissues based on TCGA (A), GSE53752 (B), GSE65216 (C) and GSE31428 (D). (E) Pathway overlap was identified using a Venn diagram in A-D. (F-I) Correlation between the expression of SUMOylation and 50 oncogenic 
pathways in TNBC normal breast tissues based on TCGA (F), GSE53752 (G), GSE65216 (H) and GSE31428 (I). (J) Pathway overlap was identified using a Venn diagram in F-I. (K) Pathway overlap was identified using a Venn diagram in TNBC tumor tissues and normal tissues.
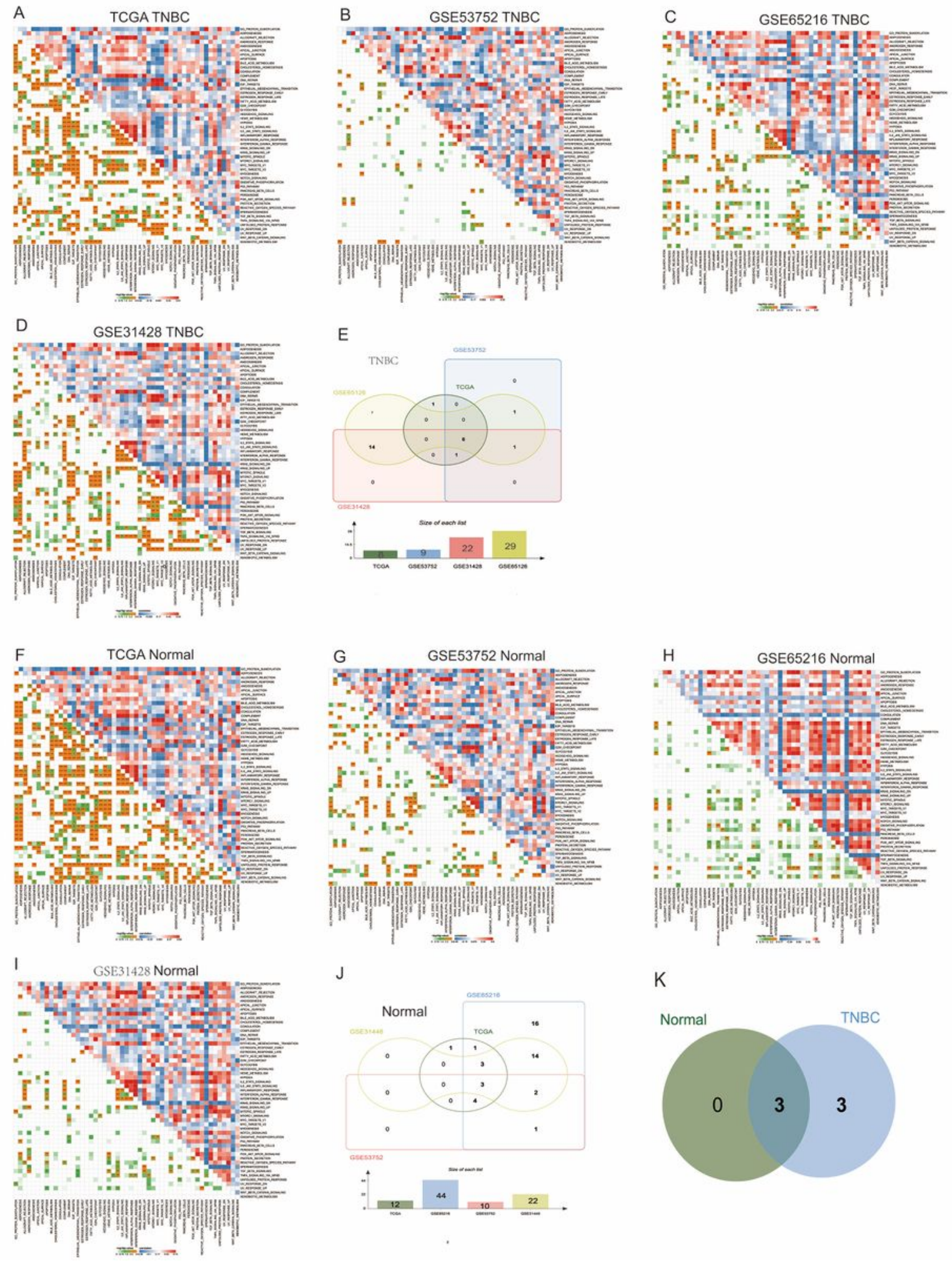

J

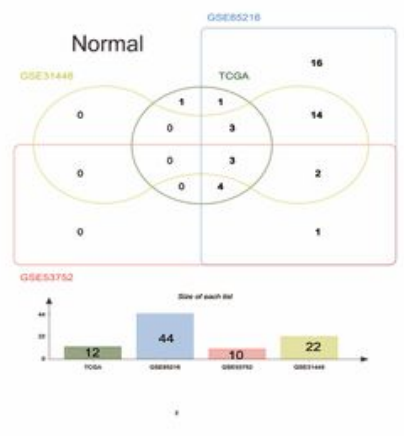

$\mathrm{K}$

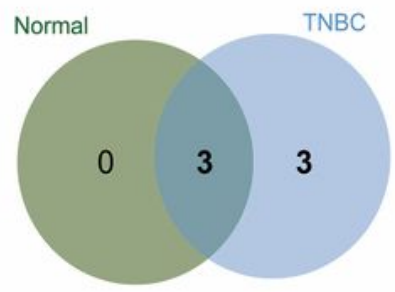

Figure 7

The positive correlation between SUMOylation and E2F-targets, MYC-targets-V1, Mtorc1-signaling, Mitotic-spindle, G2M-checkpoint and Unfolded protein response in TNBC tumor tissues (A-D) Correlation 
between the expression of SUMOylation and 50 oncogenic pathways in TNBC tumor tissues based on TCGA (A), GSE53752 (B), GSE65216 (C) and GSE31428 (D). (E) Pathway overlap was identified using a Venn diagram in A-D. (F-I) Correlation between the expression of SUMOylation and 50 oncogenic pathways in TNBC normal breast tissues based on TCGA (F), GSE53752 (G), GSE65216 (H) and GSE31428 (I). (J) Pathway overlap was identified using a Venn diagram in F-I. (K) Pathway overlap was identified using a Venn diagram in TNBC tumor tissues and normal tissues.

A

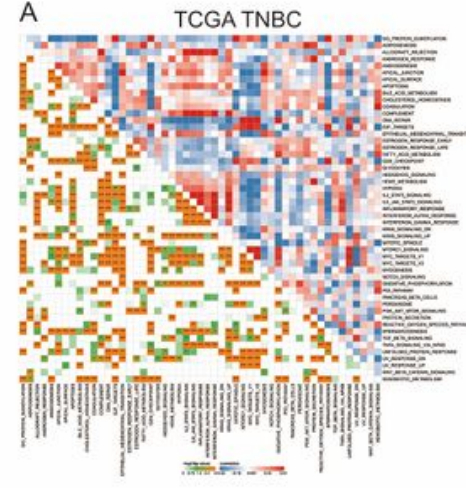

D GSE31428 TNBC

Mr prevatu= $-1,2+0=7 y=$ 4: $34=91 \mathrm{H}=$

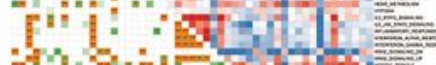
fon ; : $3: 51=4=2$ [in!
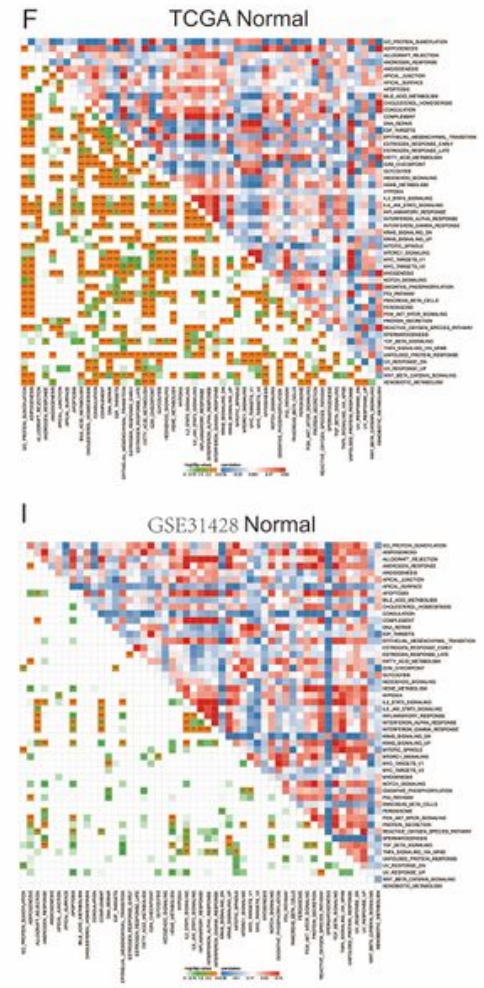

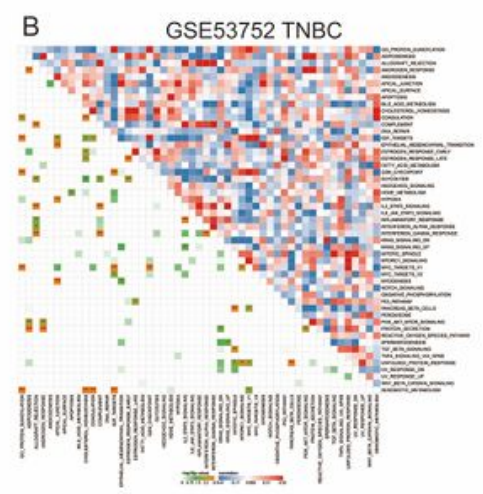

$E$

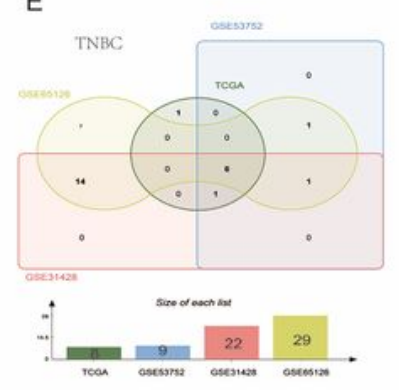

G GSE53752 Normal

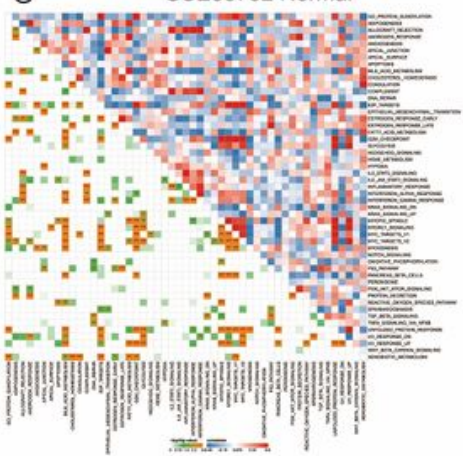

J

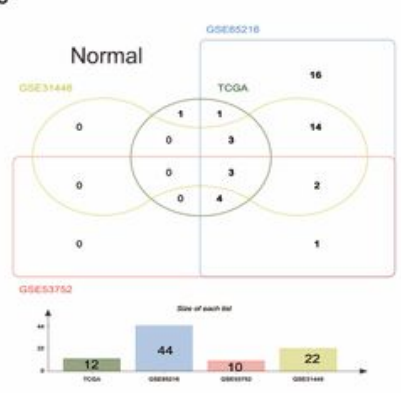

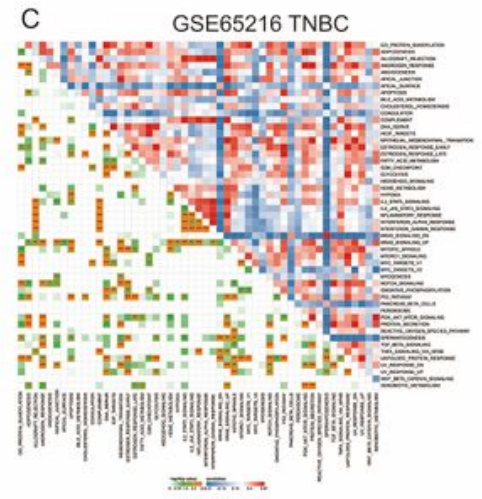

H GSE65216 Normal

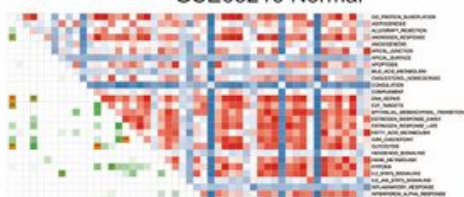

i.

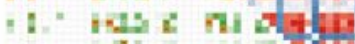

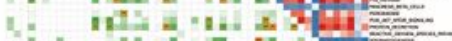

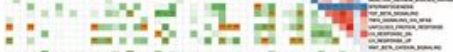

|y

K

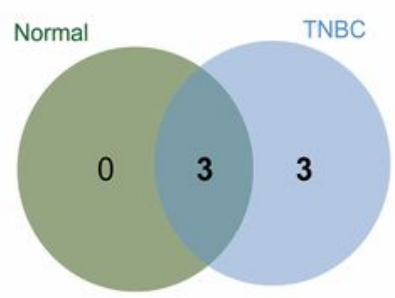


The positive correlation between SUMOylation and E2F-targets, MYC-targets-V1, Mtorc1-signaling, Mitotic-spindle, G2M-checkpoint and Unfolded protein response in TNBC tumor tissues (A-D) Correlation between the expression of SUMOylation and 50 oncogenic pathways in TNBC tumor tissues based on TCGA (A), GSE53752 (B), GSE65216 (C) and GSE31428 (D). (E) Pathway overlap was identified using a Venn diagram in A-D. (F-I) Correlation between the expression of SUMOylation and 50 oncogenic pathways in TNBC normal breast tissues based on TCGA (F), GSE53752 (G), GSE65216 (H) and GSE31428 (I). (J) Pathway overlap was identified using a Venn diagram in F-I. (K) Pathway overlap was identified using a Venn diagram in TNBC tumor tissues and normal tissues.
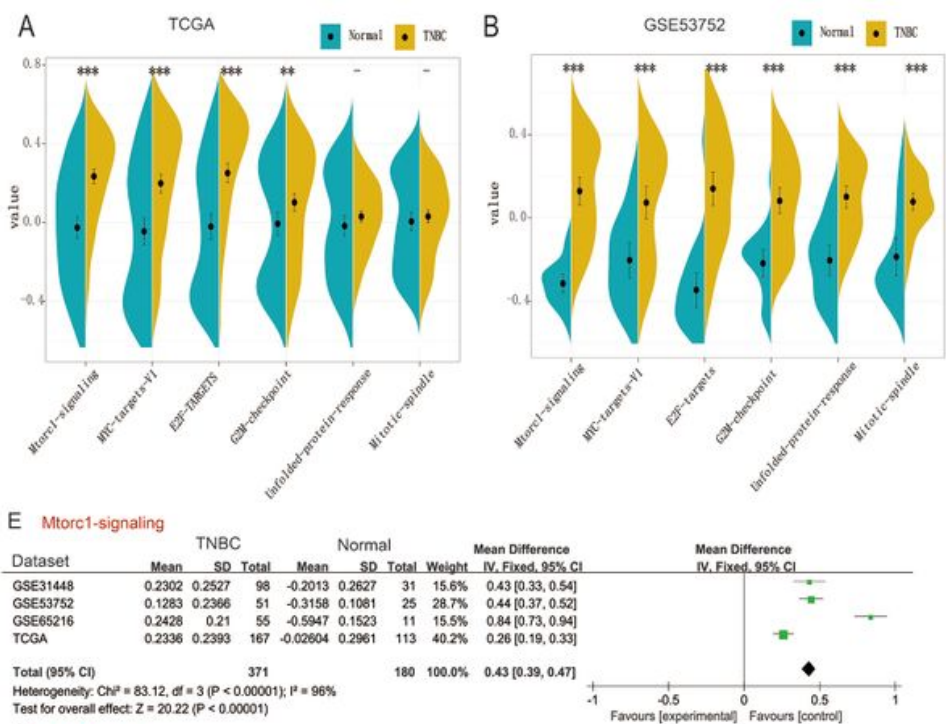

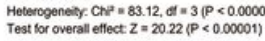

G E2F-Targets

Dataset

GSE31448

GSES375

TCGS

Total (95\% Cl)

Heterogeneity Chip $=204.74$, at $=3 . \mathrm{P}<000000$

Test for overall effect: $Z=29.75$ ( $P<0.000001)$

I Unfolded-protein-respones

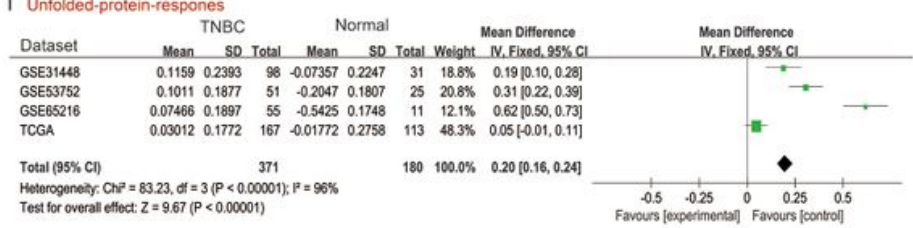

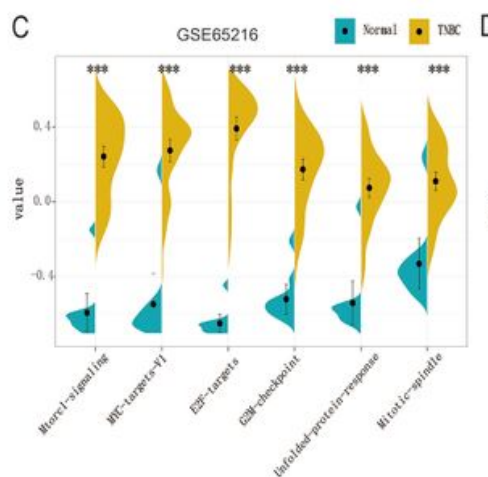
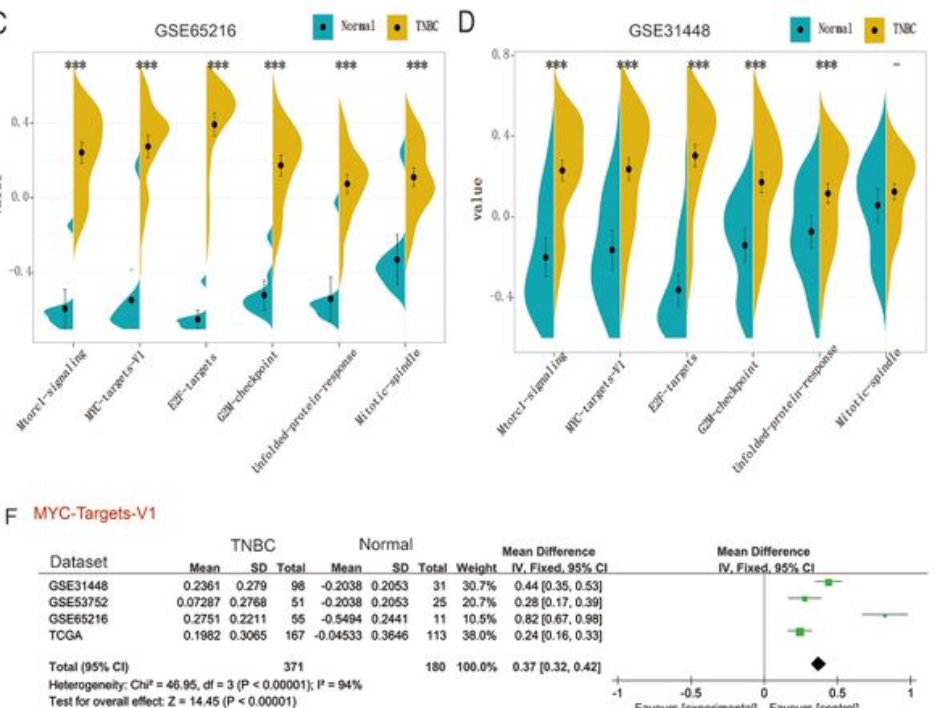

H G2M-checkpoint

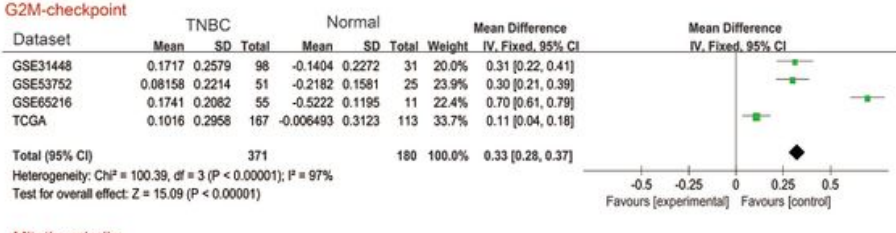

J Mitotic-spindle

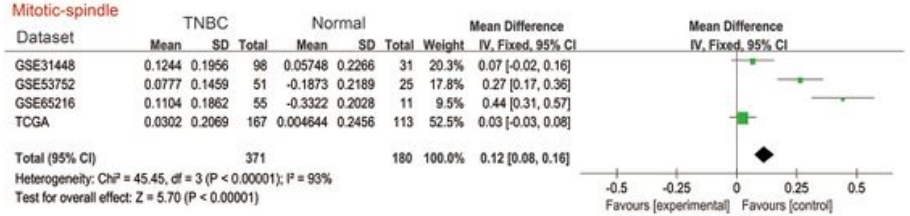

Figure 8

The pathway of E2F-targets, MYC-targets-V1, Mtorc1-signaling, Mitotic-spindle, G2M-checkpoint and Unfolded protein response are up-regulated in TNBC tumor tissues (A-D) The pathway activity of E2Ftargets, MYC-targets-V1, Mtorc1-signaling, Mitotic-spindle, G2M-checkpoint and Unfolded protein response in TNBC tumor tissues and normal breast tissue of TCGA (A), GSE53752 (B), GSE65216 (C), GSE31448 (D) based on GSVA analysis. (E-J) Meta-analysis of pathway activity of Mtorc1-signaling (E), MYC-targets-V1 (F), E2F-targets (G), G2M-checkpoint (H), Unfolded protein response (I) and Mitoticspindle $(\mathrm{J})$ in TNBC patients based on TCGA, GSE53752, GSE65216 and GSE314486. - , p>0.05; **, $\mathrm{p}<0.01$; $\star \star \star, p<0.001$. 

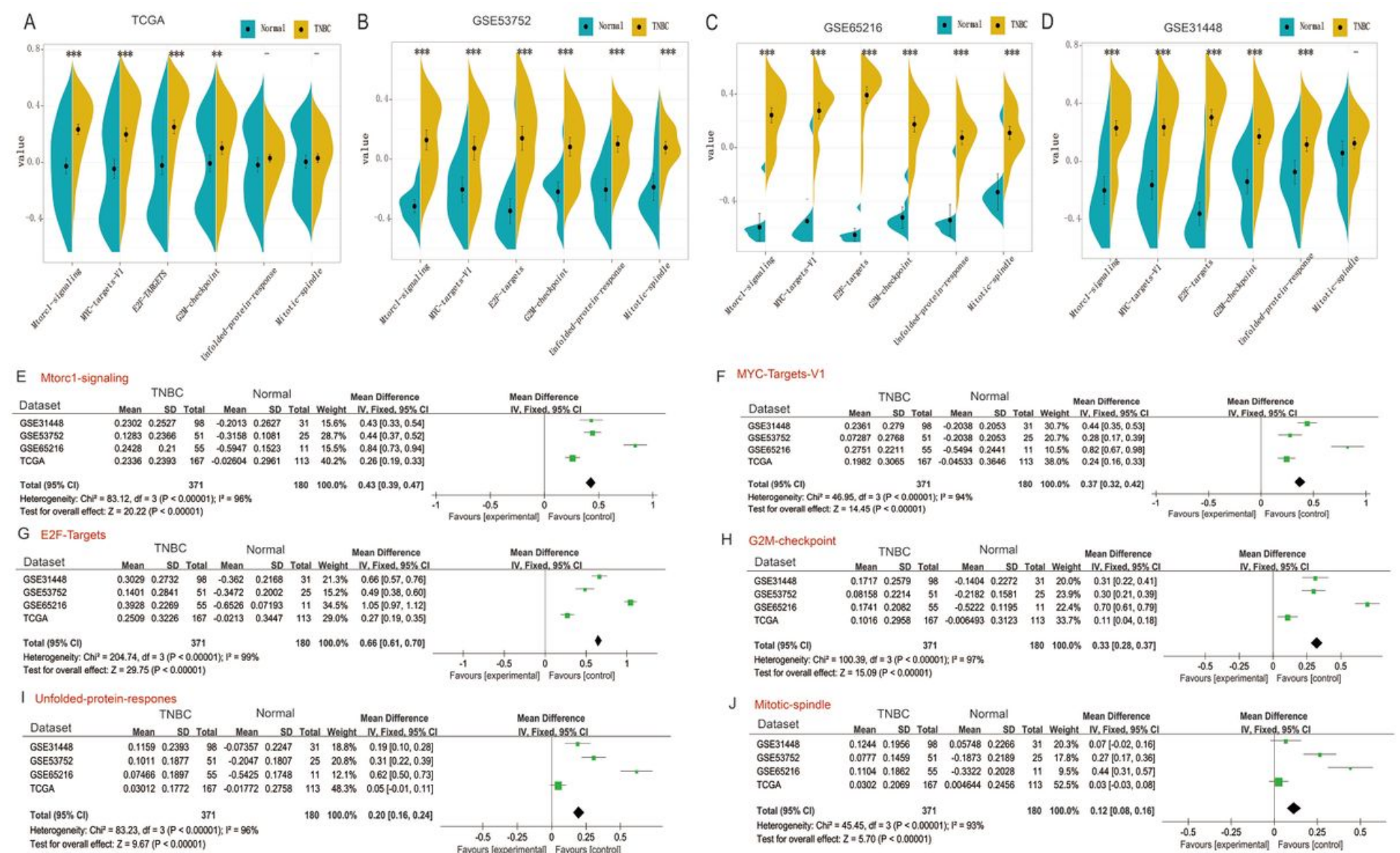

\section{Figure 8}

The pathway of E2F-targets, MYC-targets-V1, Mtorc1-signaling, Mitotic-spindle, G2M-checkpoint and Unfolded protein response are up-regulated in TNBC tumor tissues (A-D) The pathway activity of E2Ftargets, MYC-targets-V1, Mtorc1-signaling, Mitotic-spindle, G2M-checkpoint and Unfolded protein response in TNBC tumor tissues and normal breast tissue of TCGA (A), GSE53752 (B), GSE65216 (C), GSE31448 (D) based on GSVA analysis. (E-J) Meta-analysis of pathway activity of Mtorc1-signaling (E), MYC-targets-V1 (F), E2F-targets (G), G2M-checkpoint (H), Unfolded protein response (I) and Mitoticspindle $(J)$ in TNBC patients based on TCGA, GSE53752, GSE65216 and GSE314486. -, p>0.05; **, $p<0.01$; $\star \star \star, p<0.001$. 

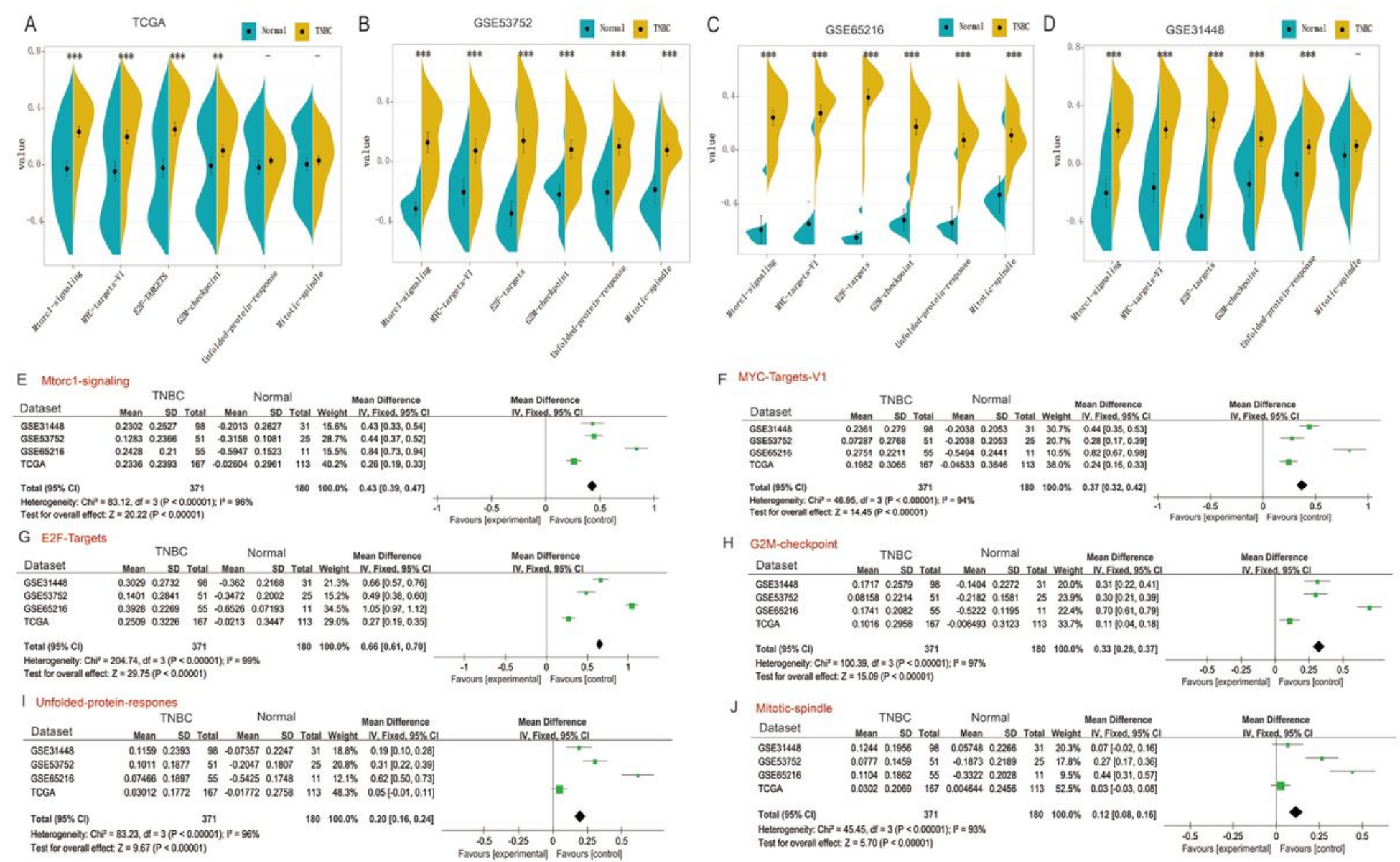

\section{Figure 8}

The pathway of E2F-targets, MYC-targets-V1, Mtorc1-signaling, Mitotic-spindle, G2M-checkpoint and Unfolded protein response are up-regulated in TNBC tumor tissues (A-D) The pathway activity of E2Ftargets, MYC-targets-V1, Mtorc1-signaling, Mitotic-spindle, G2M-checkpoint and Unfolded protein response in TNBC tumor tissues and normal breast tissue of TCGA (A), GSE53752 (B), GSE65216 (C), GSE31448 (D) based on GSVA analysis. (E-J) Meta-analysis of pathway activity of Mtorc1-signaling (E), MYC-targets-V1 (F), E2F-targets (G), G2M-checkpoint (H), Unfolded protein response (I) and Mitoticspindle (J) in TNBC patients based on TCGA, GSE53752, GSE65216 and GSE314486. -, p>0.05; **, p<0.01; $\star * \star, p<0.001$. 

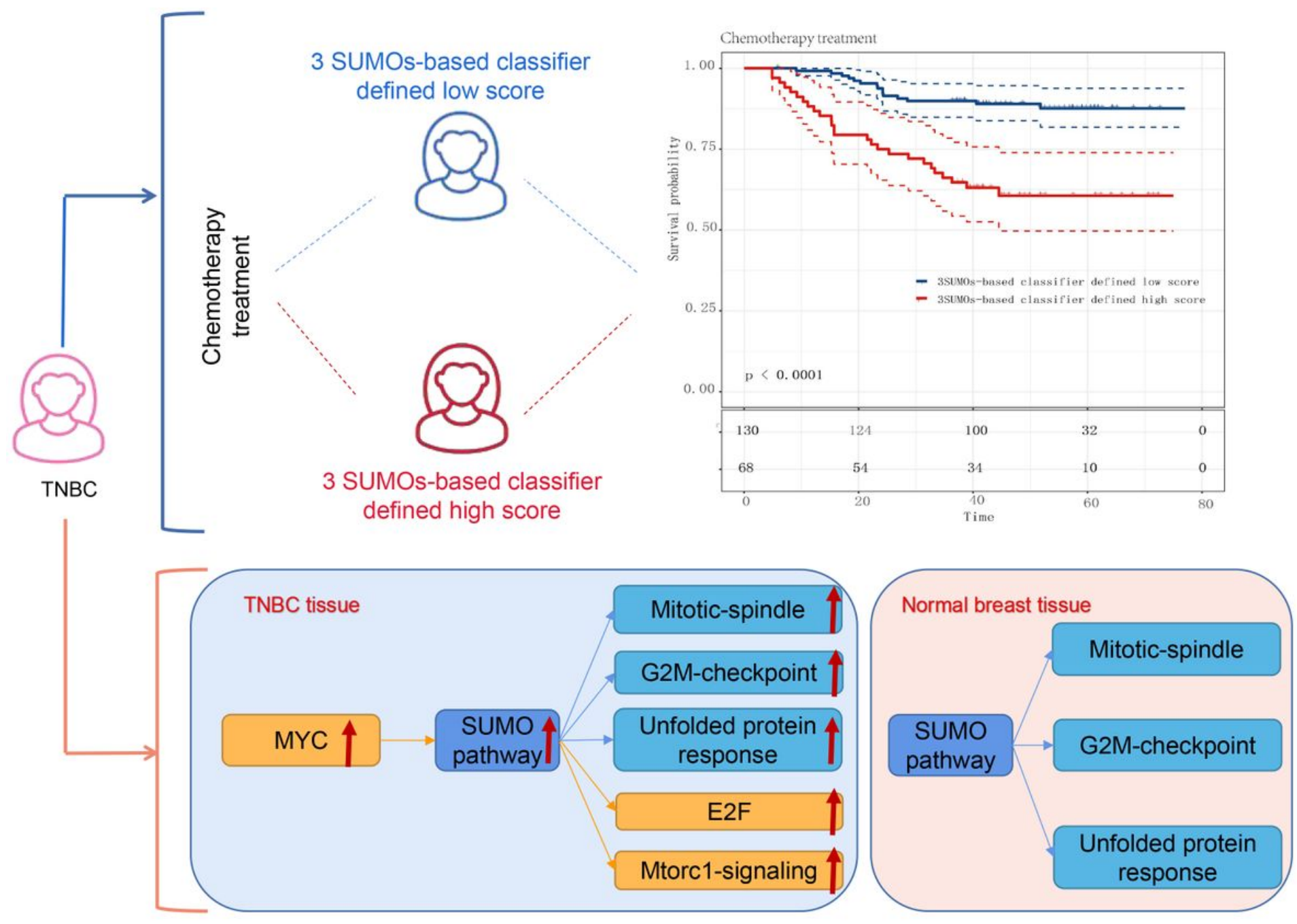

\section{Figure 9}

Pattern diagram of predicting disease free survival based on 3 SUMOs based classifier and potential mechanisms of SUMOylation regulation in TNBC 

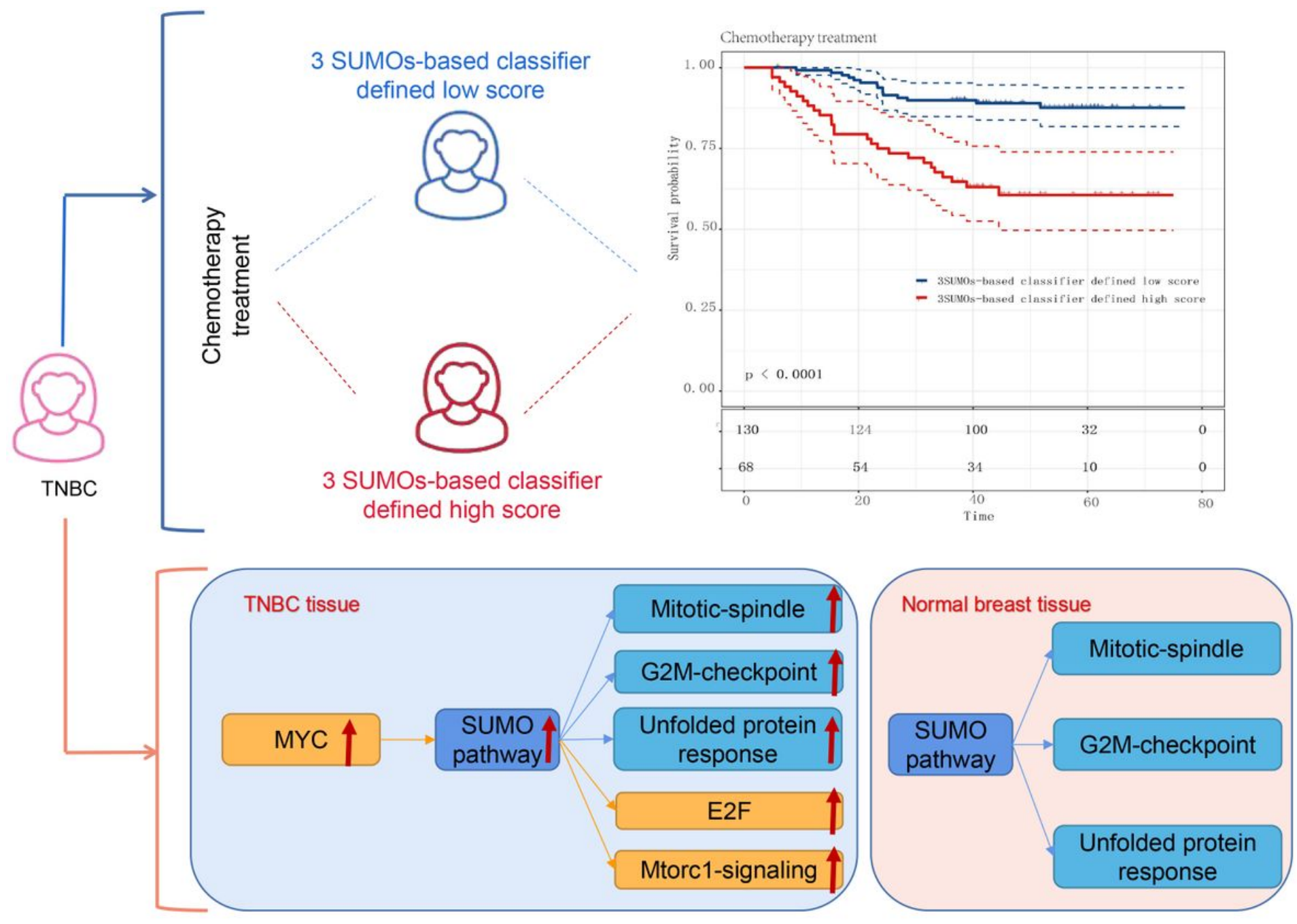

\section{Figure 9}

Pattern diagram of predicting disease free survival based on 3 SUMOs based classifier and potential mechanisms of SUMOylation regulation in TNBC 


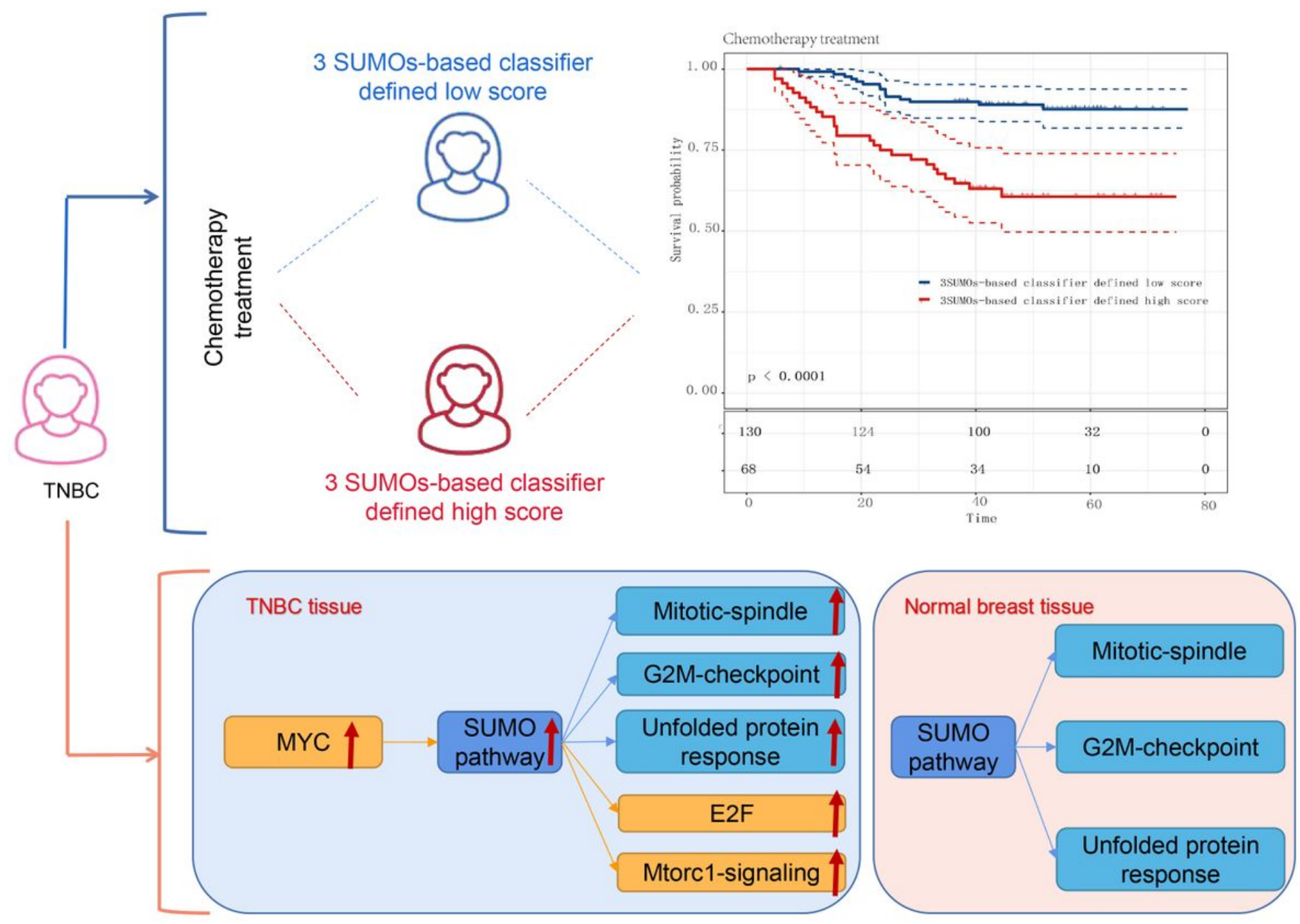

\section{Figure 9}

Pattern diagram of predicting disease free survival based on 3 SUMOs based classifier and potential mechanisms of SUMOylation regulation in TNBC

\section{Supplementary Files}

This is a list of supplementary files associated with this preprint. Click to download.

- Table1..xls

- Table1..xls

- Table1..xls

- SupplementaryTable1.xlsx

- SupplementaryTable1.xlsx

- SupplementaryTable1.xlsx 